Universidade de São Paulo

Instituto de Física

\title{
Análise Geométrica e Dinâmica de Modelos de Gravidade Generalizada
}

\author{
José Cleriston Campos de Souza
}

Orientador: Prof. Dr. Alberto Vazquez Saa

\section{Banca Examinadora}

Prof. Dr. Alberto Vazquez Saa (IMECC-Unicamp)

Prof. Dr. Adilson José da Silva (IFUSP)

Prof. Dr. Gustavo Alberto Burdman (IFUSP)

Prof. Dr. Reuven Opher (IAG-USP)

Prof. Dr. Jailson Souza de Alcaniz (ON)

Tese de doutorado apresentada ao

Instituto de Física para a obtenção

do título de Doutor em Ciências.

São Paulo

2008 


\section{Resumo}

Este trabalho teve por objetivo investigar alguns aspectos dinâmicos de modelos de gravidade generalizada escalares-tensoriais e $f(R)$, que pretendem resolver de modo mais natural o problema da existência da energia escura, que seria a componente do Universo responsável por sua expansão acelerada. Num espaço-tempo de Friedmann-LemaîtreRobertson-Walker com curvatura espacial nula foi possível escrever as equações de movimento de forma a se obter um sistema dinâmico com um número menor de variáveis e cujo espaço de fase foi estudado genericamente e esboçado para alguns modelos em particular. Em seguida, as regiões dinamicamente proibidas e os pontos fixos do espaço de fase foram analisados. Para os modelos $f(R)$, apresentamos Lagrangianas e Hamiltonianas efetivas e deduzimos uma expressão geral para o parâmetro de equação de estado $w$. Discutimos ainda a equivalência entre os modelos $f(R)$ e os escalares-tensoriais. Por fim, introduzimos o Princípio de Maupertuis-Jacobi, que permite relacionar a Lagrangiana de um sistema mecânico a uma métrica numa determinada variedade Riemanniana, para determinar singularidades que podem surgir nos modelos $f(R)$, tanto numa métrica isotrópica como numa anisotrópica do tipo mais simples (Bianchi tipo I). Encontramos, de maneira mais direta, as mesmas singularidades já conhecidas através de métodos de análise dinâmica. 


\begin{abstract}
This work aims the investigation of some dynamical aspects of generalized gravity models, namely scalar-tensor and $f(R)$ models. These models intend to solve in a more natural way the problem of the existence of the dark energy, which is supposedly the component of the Universe that causes its accelerated expansion. In a null spatial curvature Friedmann-Lemaître-Robertson-Walker spacetime, it has been possible to write the equations of movement in a fashion that allowed us to obtain a dynamical system with a reduced number of variables, whose phase space has been generically studied and depicted for some particular models. In sequence, the dynamically forbidden regions and the fixed points of the phase space have been analyzed. For $f(R)$ models, we have presented effective Lagrangians and Hamiltonians and derived a general expression for the equation of state parameter $w$. Furthermore, we have discussed the equivalence between $f(R)$ and scalar-tensor models. Finally, we have introduced the MaupertuisJacobi Principle, which allows one to relate the Lagrangian for a mechanical system to a metric in a certain Riemannian manifold, to determine singularities which may appear in $f(R)$ models, in an isotropic metric as well as in an anisotropic one of the simplest kind (Bianchi type I). We have found, in a more direct way, the same singularities that arise by using dynamical analysis methods.
\end{abstract}




\section{Agradecimentos}

Ao CNPq, pelo apoio financeiro.

Ao Prof. Dr. Alberto Saa, pela orientação, apoio e incentivo ao longo destes anos.

À Bishop's University, de Sherbrooke, Québec, Canadá, pela infraestrutura disponibilizada durante o período de um ano que lá passei, como parte do programa de doutorado-sanduíche do CNPq.

Ao Prof. Dr. Valerio Faraoni, supervisor do doutorado-sanduíche, pela hospitalidade, gentileza e apoio durante minha estadia em Sherbrooke, onde parte deste trabalho foi feita. As conversas e discussões (sobre Física e muitos outros assuntos) que tivemos resultaram numa colaboração que foi para mim muito proveitosa. Estendo meus agradecimentos também a Louine e Donovan.

Aos amigos feitos em solo canadense, em especial Veeresh Pavate, Tawhid Waez, Iram Ali e Anita Mahal. Sem seu apoio, minha adaptação em terras estrangeiras (e tão exóticas!) teria certamente sido bem mais difícil.

Aos meus senhorios, Rita Lemay e seu marido Roma (in memoriam), que me receberam com muita cortesia e paciência.

À comunidade brasileira em Sherbrooke.

Aos colegas de grupo, Fabio Cabral Carvalho, Rodrigo P. Macedo e Michele F. Figueiró.

Aos colegas do Departamento de Física Matemática (DFMA), muito numerosos para serem citados nominalmente sem algum esquecimento involuntário e injusto.

Aos amigos Marcelo O. C. Pires, Karlucio H. C. Castello Branco, Fabio Paolini, Carlos Molina Mendes e Gerson G. Gomes.

Às secretárias do DFMA, Amélia, Simone e Beth, pelo apoio estrutural.

Aos analistas do sistema de informática do departamento, Sybelle e João, pelo apoio técnico no uso dos computadores.

Aos funcionários da secretaria de pós-graduação do Instituto de Física da USP, Cláudia, Francisleine e Éber.

A Kelly C. de Carvalho, André L. Paulilo, Ricardo Martini e Luciano Moreno, amigos de longa data que, mesmo que não nos encontremos com a freqüência que gostaríamos, sempre têm uma porta aberta para mim em suas vidas, e me acompanharam, de uma forma ou de outra, ao longo de todos esse anos.

À minha família. 
A Beatriz P. Christino, Bia, pelo companheirismo, confiança, carinho e por mais do que eu consigo descrever aqui. 


\section{Conteúdo}

1 Introdução 1

2 Teorias escalares-tensoriais $\quad 8$

2.1 Modelo de Brans-Dicke . . . . . . . . . . . . . . . . . . 11

2.2 Espaço de fase do modelo de Brans-Dicke . . . . . . . . . . . . . 12

2.2.1 O caso $V=\frac{1}{2} m^{2} \phi^{2}$ e $K=0 \ldots \ldots \ldots \ldots$

$2.2 .2 \quad$ O caso $V=\Lambda \phi$ e $K=0 \ldots \ldots \ldots \ldots$

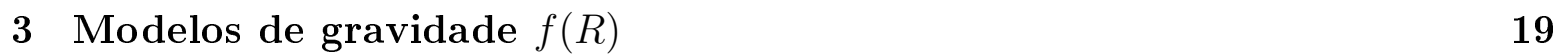

3.1 A equação de estado efetiva . . . . . . . . . . . . . . 24

3.2 A teoria escalar-tensorial equivalente . . . . . . . . . . . 25

4 Singularidades em modelos $f(R)$ anisotrópicos $\quad 28$

4.1 O Princípio de Maupertuis-Jacobi em modelos de acoplamento não-mínimo 29

4.2 Métrica anisotrópica . . . . . . . . . . . . . . . 31

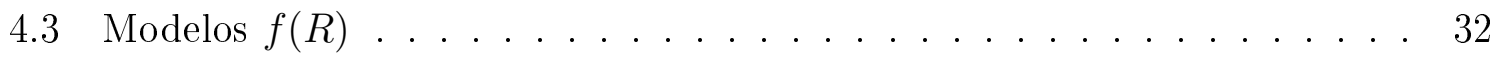

4.3.1 Métrica isotrópica . . . . . . . . . . . . . . . . 32

4.3.2 Métrica anisotrópica .................... 33

5 Conclusões e Comentários $\quad 34$

$\begin{array}{ll}\text { A Método de Lyapunov } & 37\end{array}$

B As equações de Friedmann com constante cosmológica 39

$\begin{array}{ll}\text { Bibliografia } & 41\end{array}$ 


\section{Lista de Figuras}

1.1 Diagrama de Hubble para um conjunto de supernovas. . . . . . . . . . 2

1.2 Espectro de potência da CMB. . . . . . . . . . . . . . 3

1.3 Flutuações da CMB segundo o WMAP. . . . . . . . . . . . . . . 3

1.4 Equação de estado $w(z)$ para modelos phantom. . . . . . . . . . . 5

1.5 Equação de estado $w$ para NMC. . . . . . . . . . . . . . 6

2.1 Folha superior do espaço de fase para um modelo com $\omega=10$ (Brans-Dicke). 14

2.2 Folha inferior do espaço de fase, correspondendo agora ao sinal negativo na eq. (2.2.10), com $\dot{\phi}$ no eixo vertical. . . . . . . . . . . . 15

2.3 Espaço de fase para o modelo de Brans-Dicke com $\omega=10$. . . . . . . 15

2.4 Folha superior do espaço de fase para um modelo de Brans-Dicke com $\omega=50000$ e $V=\Lambda \phi \ldots \ldots \ldots \ldots \ldots$

2.5 Folha inferior do espaço de fase, para o caso anterior. . . . . . . . . . 17

2.6 Espaço de fase completo. . . . . . . . . . . . . . . 17

$3.1 \quad$ Espaço de fase para $f(R)=R+\alpha R^{2} \ldots \ldots \ldots \ldots 21$

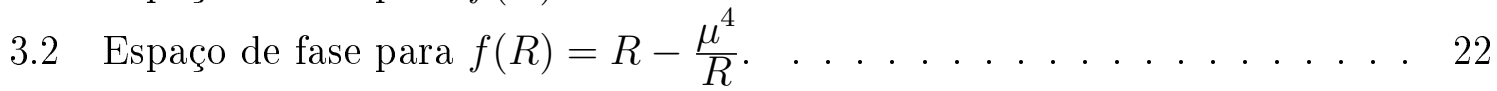

A.1 Estabilidade em sistemas dinâmicos. . . . . . . . . . . . . 38 


\section{Capítulo 1}

\section{Introdução}

Vivemos atualmente a chamada era da Cosmologia de precisão, ao mesmo tempo estimulante e desafiadora em sua capacidade de substituir por dados observacionais o que há bem pouco tempo era apenas especulação.

Apesar disso, é um engano pensar que os conhecidos problemas das teorias cosmológicas (desde o seu surgimento, com os trabalhos de Einstein e Friedmann) estejam resolvidos a partir do momento em que se possam ajustar observações a algum método estatístico independente de um modelo teórico. Surge, então, o problema da escolha do modelo cosmológico mais adequado dos pontos de vista físico, matemático e observacional. Esta é uma tarefa das mais difíceis, à qual se tem dedicado uma boa parte dos físicos teóricos em atividade.

Recentemente, como resultado de medições precisas do redshift e da luminosidade de supernovas tipo Ia efetuadas com o Telescópio Espacial Hubble (ver, por exemplo, [81]) e a partir de telescópios terrestres [82], descobriu-se que o Universo está passando por um período de expansão acelerada [83, 25, 26]. A figura 1.1 mostra o diagrama de Hubble para um desses conjuntos de medidas.

As equações de Einstein requerem um fluido cosmológico com pressão negativa agindo como fonte para uma fase de expansão acelerada do Universo. Tal fluido repulsivo deve ser não-luminoso, ou de outra forma já teria sido detectado. Ele representa a componente de energia "escura" que domina a evolução do Universo hoje e corresponde a $70 \%$ da composição do Universo.

As observações das flutuações na radiação cósmica de fundo (CMB), principalmente os espetaculares resultados do WMAP [85](ver as figuras 1.2 e 1.3), reforçaram a hipótese da energia escura. Apesar dos graves problemas de degenerescência na análise dos 


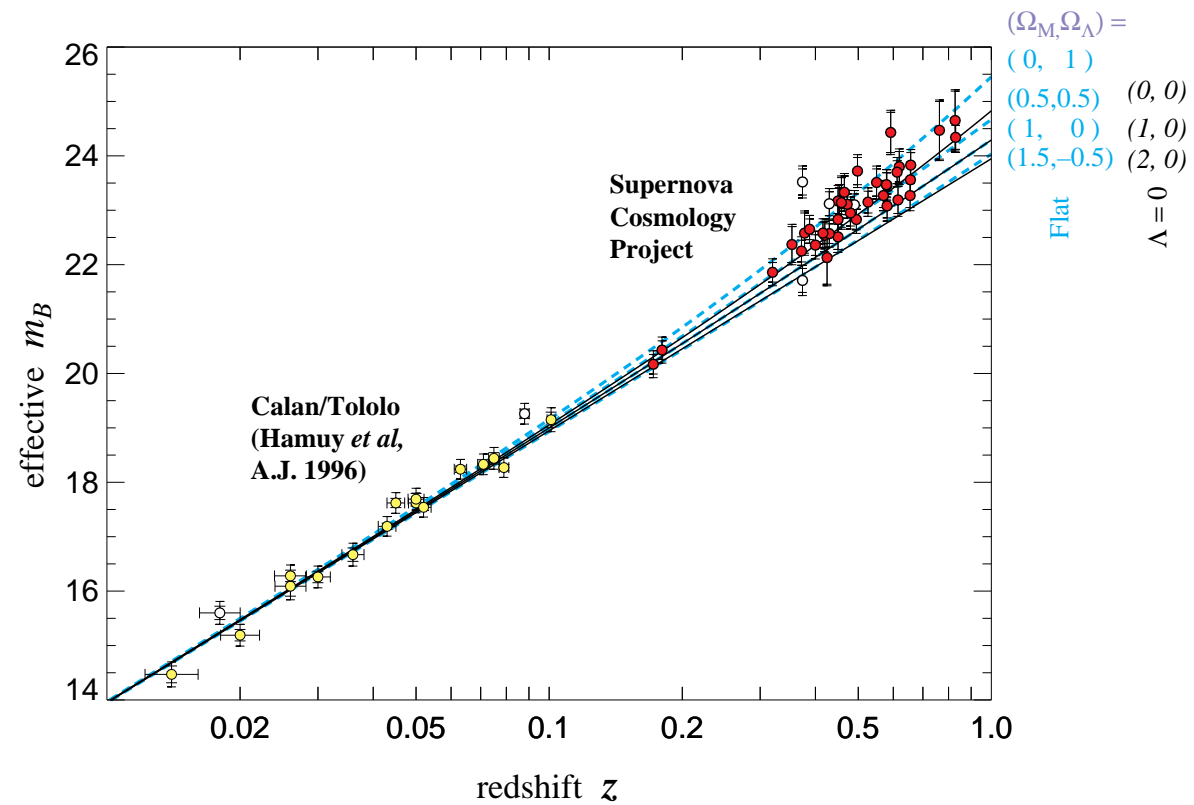

Figura 1.1: Diagrama de Hubble para um conjunto de supernovas. As supernovas mais distantes são menos brilhantes (maior magnitude aparente $m_{B}$ ) do que o esperado para um Universo em que valesse a lei linear de Hubble em larga escala. São mostradas as curvas correspondentes a alguns parâmetros cosmológicos diferentes, dentre as quais as que melhor se ajustam fazem uso de uma componente de energia escura $\left(\Omega_{\Lambda}\right)$, especialmente para redshifts mais altos. (Extraído de [25].)

parâmetros da CMB, a existência de uma componente de energia escura representada por uma pequena constante cosmológica $\Lambda$ é tão favorecida pelos dados disponíveis que o modelo cosmológico padrão recebe o nome de modelo $\Lambda$ CDM, sendo CDM Cold Dark Matter, matéria escura fria, uma forma de matéria não-luminosa gravitacionalmente atrativa, responsável por aproximadamente $25 \%$ do conteúdo de energia do Universo.

Entretanto, são bem conhecidos os problemas associados à proposição de uma constante cosmológica pequena como a origem da energia escura [87]. Por exemplo, a densidade de energia da constante cosmológica pode ser estimada usando-se métodos da teoria quântica de campos, resultando no valor

$$
\rho_{\Lambda} \sim\left(10^{18} \mathrm{GeV}\right)^{4} \sim 2 \times 10^{110} \mathrm{erg} / \mathrm{cm}^{3},
$$

enquanto as observações implicam em

$$
\rho_{\Lambda}^{\text {obs }} \sim\left(10^{-12} \mathrm{GeV}\right)^{4} \sim 2 \times 10^{-10} \mathrm{erg} / \mathrm{cm}^{3} .
$$




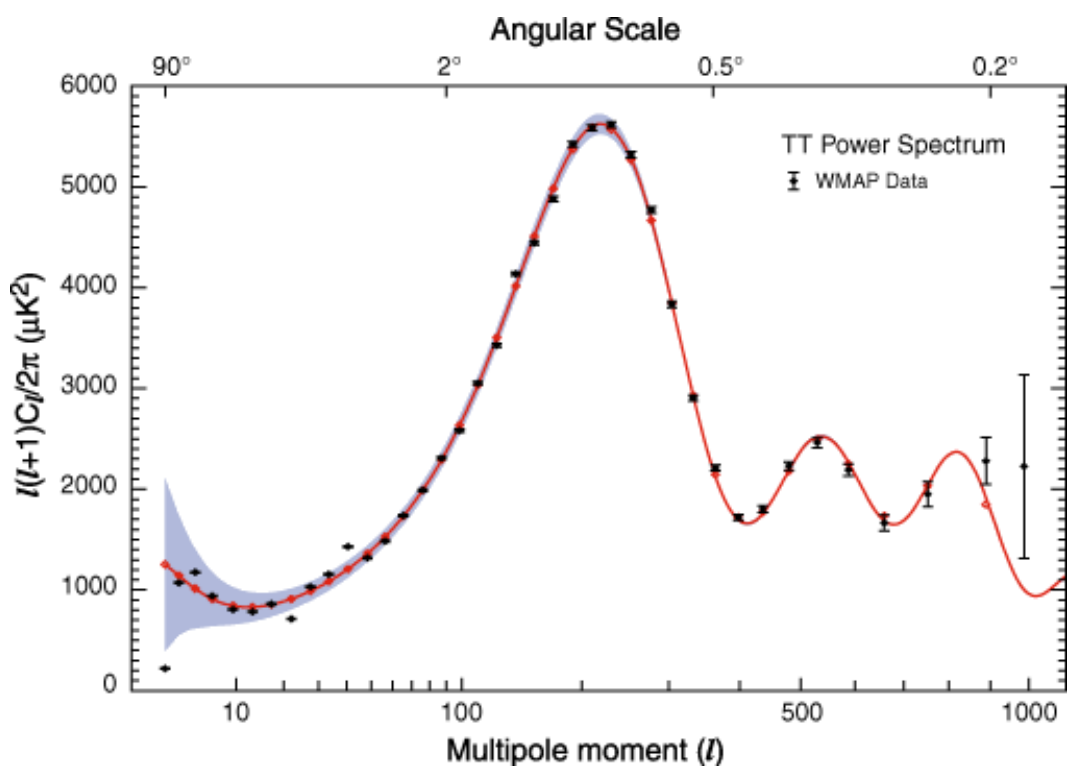

Figura 1.2: Espectro de potência da radiação cósmica de fundo, segundo dados do WMAP.

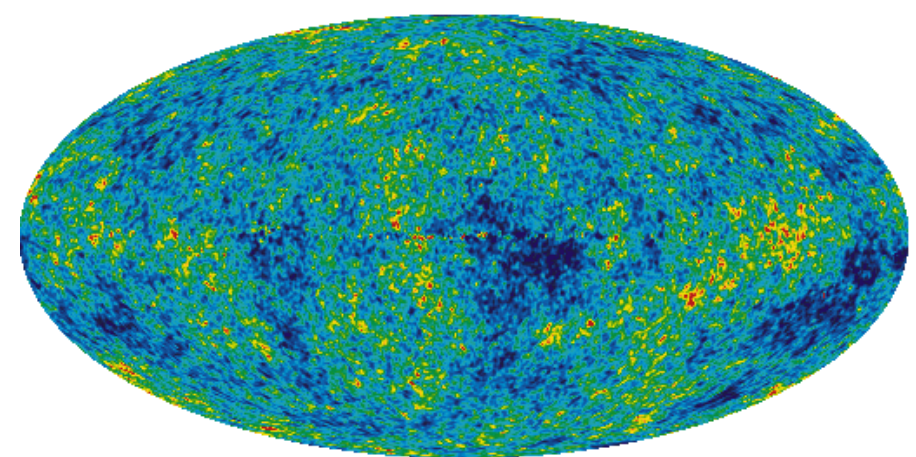

Figura 1.3: Imagem das flutuações de temperatura do Universo primordial, segundo os dados de cinco anos de coleta do WMAP.

Esta é a mais grave discrepância de toda a Física e ainda não tem solução. Assim, é justificável procurar uma teoria que prescinda de uma constante cosmológica para dar conta das observações atuais.

Como uma maneira de nos orientarmos na busca por teorias mais aceitáveis, podemos procurar parâmetros observacionais. Com esta intenção, é útil definir o chamado parâmetro de equação de estado $w$, que relaciona a densidade de energia $\rho$ e a pressão $p$ do fluido que compõe o Universo da seguinte forma

$$
w \equiv \frac{p}{\rho}
$$


Este parâmetro está ligado à velocidade de expansão do Universo através da expressão

$$
\frac{\ddot{a}}{a}=-\frac{4}{3} \pi G(\rho+3 p)=-\frac{4}{3} \pi G \rho(1+w),
$$

obtida a partir das equações de Friedmann para um Universo plano de FriedmannLemaître-Robertson-Walker, e onde $a$ é o fator de escala que define as relações de distância entre os pontos do espaço (ver Apêndice B).

Em geral, a densidade de energia do fluido que domina a constituição do Universo em uma determinada fase varia em relação ao fator de escala de acordo com

$$
\rho \propto a^{-3(1+w)},
$$

e a variação temporal do fator de escala para o fluido que domina a expansão do Universo se dá através da relação

$$
a \propto t^{-\frac{2}{3(1+w)}}
$$

Para um Universo dominado por uma constante cosmológica, entretanto, $w=-1 \mathrm{e}$ temos que $a \propto \mathrm{e}^{H t}$ (expansão de de Sitter). Quando $-1<w<-1 / 3$, o campo escalar responsável pela aceleração é chamado de quintessência.

Embora ainda dependentes de refinamentos observacionais e estatísticos, medidas recentes sugerem a possibilidade de que o parâmetro de equação de estado $w$ seja menor do que -1 (e ao mesmo tempo favorecem a situação em que $w \approx-1$, correspondendo a uma expansão do tipo quasi-de Sitter). O fato de $w$ poder ser menor que -1 leva à violação das condições de conservação de energia. Apesar disso, é possível (e muitas vezes útil) implementar modelos teóricos em que $w<-1$ através do uso de um campo escalar $\phi$, conhecido como phantom, com um potencial $V(\phi)$. Um modelo desse tipo poderia ter uma ação gravitacional da forma

$$
S=\int d^{4} x \sqrt{-g}\left[\frac{1}{16 \pi G} R+\frac{1}{2} g^{\mu \nu} \partial_{\mu} \phi \partial_{\nu} \phi-V(\phi)\right],
$$

onde $G$ é a constante de gravitação de Newton e $R$ é o escalar de curvatura.

A energia cinética desse campo, de forma não usual, seria negativa $\left(-\dot{\phi}^{2} / 2\right)$, o que permitiria escrever sua densidade de energia $\rho$ e pressão $p$ como (desde que o campo seja homogêneo)

$$
\rho=-\frac{\dot{\phi}^{2}}{2}+V(\phi) ; p=-\frac{\dot{\phi}^{2}}{2}-V(\phi) .
$$

Agora, o parâmetro de equação de estado pode ser definido por

$$
w \equiv \frac{p}{\rho}=\frac{\frac{1}{2} \dot{\phi}^{2}+V(\phi)}{\frac{1}{2} \dot{\phi}^{2}-V(\phi)}
$$


e satisfaz $w \leq-1$.

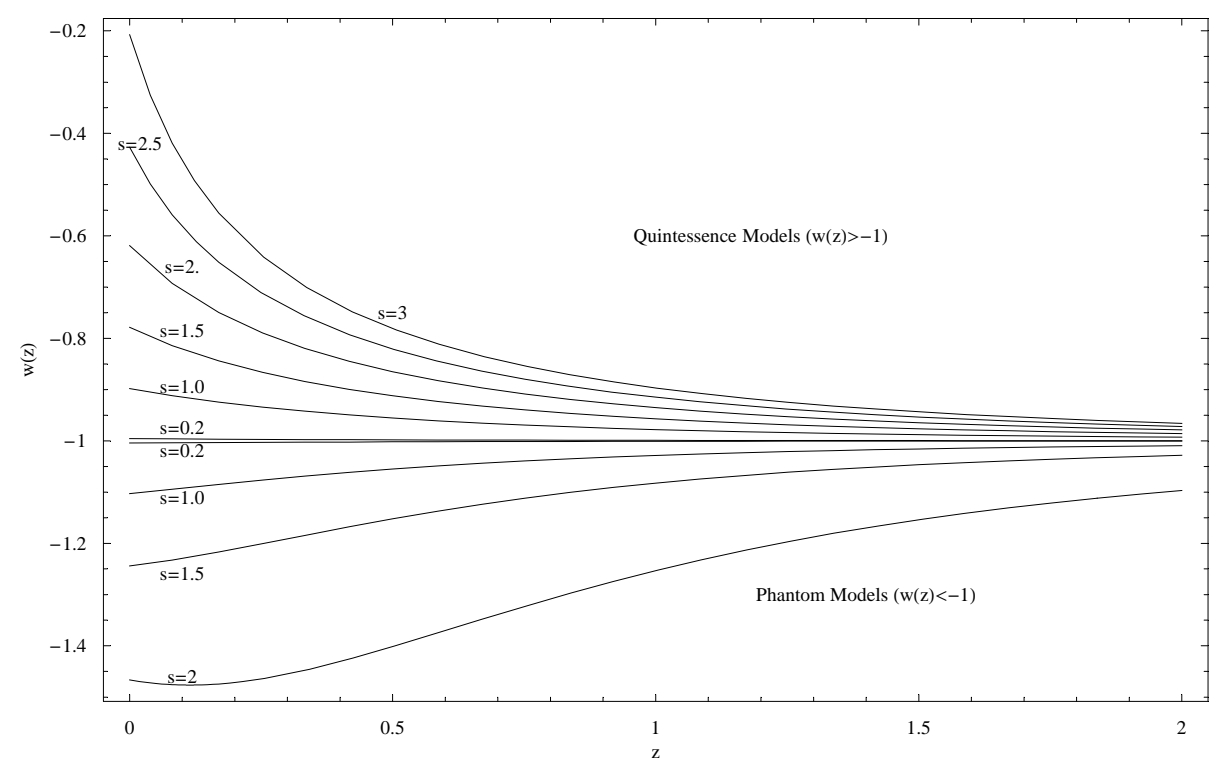

Figura 1.4: Parâmetro de equação de estado $w(z)$ para vários modelos phantom e de quintessência com potenciais ligeiramente diferentes $(V(\phi)=s \phi)$. A linha $w(z)=-1$ é a chamada phantom divide line, que corresponde a um modelo dotado de constante cosmológica. (Extraído de [77].)

$\mathrm{Na}$ verdade, a equação de estado não é necessariamente uma constante, mas pode depender do tempo, e, portanto, do redshift z. É possível inclusive que ela tenha cruzado a phantom divide line, a linha correspondente a uma constante cosmológica, $\operatorname{com} w=-1$ (ver figura 1.4). Para uma descrição teórica mais adequada da evolução do Universo, é importante procurar uma parametrização de $w(z)$ que seja coerente com as observações já realizadas ou em fase de planejamento.

Por apresentarem problemas de instabilidade tanto em nível clássico como quântico, os modelos phantom são considerados inviáveis para a descrição do Universo primordial, e soluções alternativas para explicar esse valor do parâmetro de estado têm sido investigadas, como, por exemplo, o potencial exponencial estudado em [62]. Nesse trabalho, mostra-se que modelos não-minimamente acoplados apresentam fases de de Sitter assin- 
tóticas para vários conjuntos de condições iniciais, algumas das quais se caracterizam por um parâmetro $w<-1$, como indicam as observações, mas são livres das instabilidades associadas aos modelos tipo phantom, sendo assim mais realísticos (figura 1.5).

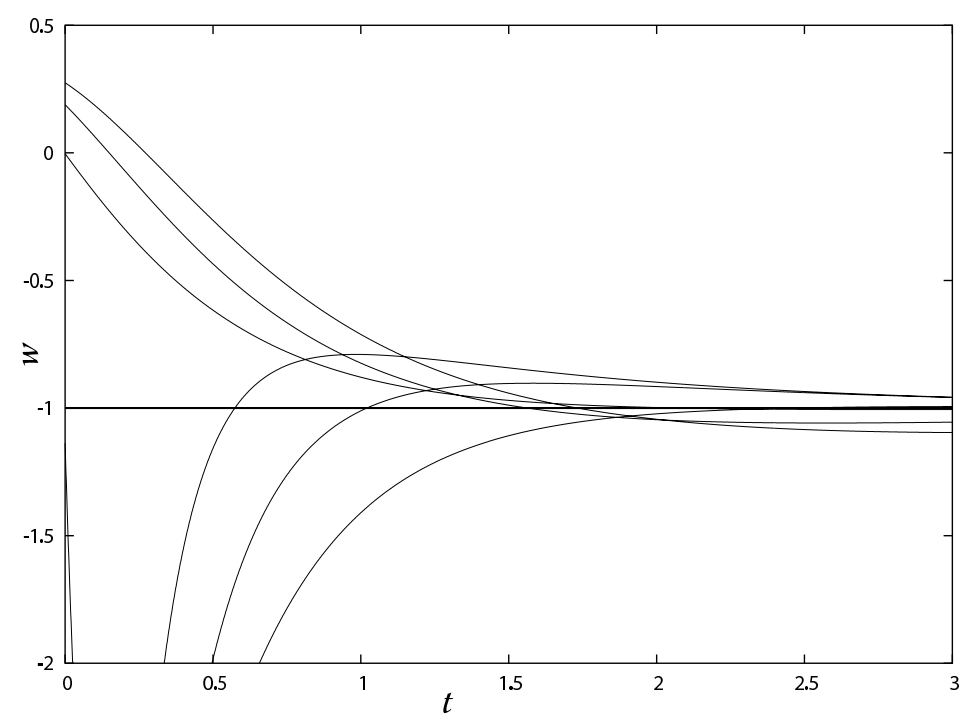

Figura 1.5: Evolução do parâmetro de equação de estado $w$ para algumas das soluções do modelo não-minimamente acoplado, com potencial exponencial. (Extraído de [62].)

A existência de uma componente de energia escura tem consequências cruciais para o que podemos esperar para a evolução do Universo. Dependendo da densidade de energia dessa componente, em relação à densidade total de energia, a expansão do Universo pode continuar eternamente a uma taxa constante, acelerar de modo muito acentuado levando a uma singularidade futura ou diminuir de tal forma a provocar uma contração.

Como alternativas à RG, numa escala cosmológica, foram propostos modelos de gravidade generalizada, que implicam numa modificação na ação de Einstein-Hilbert de forma a incluir uma constante cosmológica dinâmica ou algum tipo de campo escalar que esteja provocando a superaceleração do Universo. Como exemplos de modelos alternativos podemos citar modelos escalares-tensoriais [11], gravidade $f(R)$ e gravidade induzida. Qualquer um desses modelos deve ter a capacidade de passar também pelo crivo observacional das interações gravitacionais de escala relativamente curta do Sistema Solar (Bertotti e colaboradores [44], por exemplo, fornecem um tipo de observação que pode ser efetuada para vincular parâmetros cosmológicos).

É útil salientar que existem também outras possíveis soluções para o problema da superaceleração envolvendo, por exemplo, modelos de branas [78]. Algumas teorias 
descartam completamente a ocorrência do big bang, como a Cosmologia de cordas e os modelos ekpiróticos. Essas alternativas ainda estão sendo alvo de muitos debates e não se sabe até que ponto elas são comprováveis experimentalmente.

O próximo passo natural na busca por uma alternativa viável ao modelo padrão é construir modelos realísticos e comparar suas predições com os ricos dados observacionais já disponíveis. Muitas hipóteses estarão em condições de serem testadas experimentalmente, muitos modelos serão refutados e o nosso conhecimento sobre a natureza da energia escura será certamente aumentado. Num futuro próximo, esses modelos terão vínculos ainda mais fortes através de medidas de experimentos como o SNAP [86, 75].

Acreditamos que ainda há um longo caminho a percorrer antes que se tenha fundamentado uma nova teoria física que seja satisfatória como uma explicação para os fenômenos que se tem observado e ao mesmo tempo seja profunda o suficiente para englobar outras teorias já estabelecidas, esclarecendo conceitos e fazendo previsões testáveis.

Como um dos aspectos fundamentais de qualquer modelo cosmológico realístico é o seu comportamento dinâmico, o presente trabalho visa dar uma contribuição a esse esforço de pesquisa, apresentando um estudo de modelos alternativos de gravitação (em especial, de modelos escalares-tensoriais e de modelos $f(R)$ ) tendo como guia o método de análise dinâmica do espaço de fase. Temos como objetivo obter para esses modelos o máximo possível de informação matemática e física, antes de buscar o ajuste de parâmetros observacionais.

Para apresentar os estudos realizados, este trabalho foi estruturado em mais quatro capítulos.

No capítulo 2, descrevemos as teorias escalares-tensoriais e fazemos uma aplicação do método de análise dinâmica para alguns casos simples, esboçando o espaço de fase.

No capítulo 3, introduzimos os modelos de gravidade generalizada $f(R)$, apresentando também uma descrição geral do seu espaço de fase e ressaltando sua equivalência com os modelos escalares-tensoriais, além de deduzirmos a forma geral da equação de estado $w$ para esses modelos e também suas Lagrangianas e Hamiltonianas efetivas.

No capítulo 4, fazemos uma breve introdução ao Princípio de Maupertuis-Jacobi e o utilizamos para obter singularidades isotrópicas e anisotrópicas em modelos escalarestensoriais e $f(R)$.

Por fim, o capítulo 5 apresenta as conclusões e comentários finais.

As notações adotadas serão explicitadas conforme seu uso nas expressões. 


\section{Capítulo 2}

\section{Teorias escalares-tensoriais}

A Relatividade Geral (RG) é uma das teorias de maior sucesso já criadas. Sua capacidade de descrever muitos fenômenos físicos com uma precisão admirável, aliada a um robusto arcabouço matemático, faz dela um dos paradigmas da ciência do século XX, tanto em relação à sua profundidade como à sua elegância. É bem conhecido, até pelo público leigo, o êxito da RG no cálculo preciso da quantidades que descrevem fenômenos como o desvio da luz das estrelas pelo Sol e a precessão do periélio da órbita de Mercúrio, por exemplo. No entanto, ela também apresenta limitações envolvendo sua aplicabilidade em certas situações em que é necessário considerar escalas extremas. No caso de pequenas escalas, surge a dificuldade de se efetuar uma quantização coerente da teoria. Em escala cosmológica, a expansão acelerada do Universo atual não é explicada pela RG.

Um modo de se tentar descrever com mais acurácia as características que observamos para o Universo é alterar a RG de tal forma a se obter um formalismo similar (ou seja, a partir do qual seja possível deduzir equações de movimento e de conservação para quantidades mensuráveis) partindo de uma ação modificada. Como uma alternativa, pode-se lançar mão das chamadas teorias escalares-tensoriais [11]. Tais modelos têm-se tornado de interesse crescente por oferecer uma descrição mais apropriada do cenário inflacionário de evolução do Universo [21, 22], o qual soluciona de forma mais natural os problemas do horizonte, da planicidade e da homogeneidade do Universo, além de resolver a questão da não-detecção de monopólos magnéticos.

O estudo das flutuações de densidade geradas pela inflação primordial é importante para a investigação da formação de grandes estruturas.

As teorias de gravitação escalares-tensoriais (STT, de scalar tensor theories) podem fornecer uma constante cosmológica $\Lambda$ que é dependente do tempo, mas que para $t \gg 0$ 
torna-se uma constante independente do tempo, providenciando assim uma solução para o problema da "saída graciosa" da expansão inflacionária [27]. Elas podem oferecer também valores para os parâmetros físicos que são mais coerentes com as observações, como no caso da densidade de massa bariônica do Universo $\Omega_{b}$ (ver apêndice B). Além disso, é possível que a origem da aceleração atual do Universo [25, 26] esteja em algum tipo de campo escalar cuja densidade de energia se tornou predominante apenas em épocas recentes da história do Universo.

Outra motivação para o interesse em STT vem do fato de que teorias de supercordas envolvem um campo escalar (chamado dilaton, nesse contexto) acoplado à curvatura na sua ação efetiva [113].

Nos modelos escalares-tensoriais, a gravitação é descrita por uma ação do tipo [31]

$$
S=\frac{1}{16 \pi G_{*}} \int d^{4} x \sqrt{-g}\left[F(\Phi) R-Z(\Phi) g^{\mu \nu} \partial_{\mu} \Phi \partial_{\nu} \Phi-2 U(\Phi)\right]+S_{m}\left[\psi_{m} ; g_{\mu \nu}\right]
$$

onde $G_{*}$ é a constante de acoplamento gravitacional nua, $R$ é a curvatura escalar da métrica $g^{\mu \nu}$ e $g$ é o seu determinante. A ação de matéria $S_{m}$ é função apenas de um campo material $\psi_{m}$ e da métrica. $F(\Phi)$ e $Z(\Phi)$ são funções arbitrárias do campo escalar $\Phi$, que devem ser parametrizadas de forma a produzir uma série de teorias escalares-tensoriais. Por exemplo, na teoria usual de Brans-Dicke (seção 2.1), $F(\Phi)=\Phi$ e $Z(\Phi)=\omega(\Phi) / \Phi$, sendo $\omega$ o chamado parâmetro de Brans-Dicke. As equações de movimento são obtidas da forma usual através da variação da ação (2.0.1). Note-se que tudo isso é definido no chamado frame de Jordan, considerado um referencial mais físico que o frame de Einstein ${ }^{1}$. O frame de Jordan se relaciona com o de Einstein através da transformação conforme

$$
g_{\mu \nu}^{*} \equiv F(\Phi) g_{\mu \nu}
$$

A ação se torna agora

$$
S=\frac{1}{4 \pi G_{*}} \int d^{4} x \sqrt{-g^{*}}\left[\frac{R_{*}}{4}-\frac{1}{2} g_{*}^{\mu \nu} \partial_{\mu} \varphi \partial_{\nu} \varphi-V(\varphi)\right]+S_{m}\left[\psi_{m} ; A^{2}(\varphi) g_{\mu \nu}^{*}\right],
$$

\footnotetext{
${ }^{1}$ A questão sobre qual dos dois frames é realmente o mais físico ou o mais adequado para o estudo de problemas cosmológicos, que de há muito vem suscitando discussões e controvérsias, não será debatida aqui. Seguimos a opinião expressa em grande parte dos trabalhos a respeito desse assunto: a de que o frame de Jordan é o mais físico. Para uma discussão sobre a equivalência entre os dois frames, ver [96].
} 
onde $\varphi$ é a nova variável, e definimos

$$
\begin{aligned}
\left(\frac{d \varphi}{d \Phi}\right)^{2} & \equiv \frac{3}{4}\left(\frac{d \ln F(\Phi)}{d \Phi}\right)^{2}+\frac{Z(\Phi)}{2 F(\Phi)} \\
A(\varphi) & \equiv F^{-1 / 2}(\Phi) \\
2 V(\varphi) & \equiv U(\Phi) F^{-2}(\Phi) .
\end{aligned}
$$

O estudo das singularidades que podem surgir nos dois tipos de frames é de grande relevância, bem como o estabelecimento de uma teoria de perturbações no frame de Jordan.

É possível descrever uma variedade de teorias de gravitação a partir de uma generalização dos modelos escalares-tensoriais. Para citar um exemplo, [41] apresenta uma formulação de fluido imperfeito para equações de perturbações cosmológicas em teorias de gravitação generalizadas, a partir de uma Lagrangiana generalizada da forma (notação do autor)

$$
\beta L=\frac{1}{2} f(\phi, R)-\frac{1}{2} \omega \phi_{, \nu} \phi^{; \nu}+\beta L_{M},
$$

onde $\beta$ é uma constante necessária para ajustar as unidades e $L_{M}$ é a Lagrangiana de matéria. Esta Lagrangiana é bastante geral e inclui outras teorias como casos especiais. Por exemplo, teorias escalares-tensoriais generalizadas apresentam $f=2 \phi[R+2 \lambda(\phi)]-$ $2 V(\phi), \beta=16 \pi$ e $\omega \rightarrow 2 \omega(\phi) / \phi$, de forma que

$$
16 \pi L=\phi(R+2 \lambda)-V-\omega \frac{\phi_{, \nu} \phi^{; \nu}}{\phi}+16 \pi L_{M} .
$$

A teoria de Brans-Dicke é obtida quando $\lambda=V=0$. No caso em que $f=\alpha R-\xi \phi^{2} R-$ $2 V(\phi), \omega=1$ e $\beta=1$, temos

$$
L=\frac{1}{2} \alpha R-\frac{1}{2} \xi \phi^{2} R-V-\frac{1}{2} \phi_{, \nu} \phi^{; \nu}+L_{M} .
$$

Para $\alpha=1$, temos o caso do campo escalar não-minimamente acoplado. $\xi=0$ é o caso minimamente acoplado e, se $\alpha=0$ com um potencial específico, esta Lagrangiana representa o modelo de gravidade induzida. Ainda podemos fazer, para a Lagrangiana (2.0.7), $f=f(R), \omega=0$ e $\beta=1$, obtendo a chamada gravidade $f(R)$ :

$$
L=\frac{1}{2} f(R)+L_{M}
$$

O caso de gravidade $R^{2}$ é obtido quando $f=R-R^{2} / 6 M^{2}$. 
A gravidade $f(R)$ será investigada no próximo capítulo, com uma discussão sobre sua equivalência em relação aos modelos escalares-tensoriais sendo apresentada na seção 3.2 .

Na próxima seção introduziremos o mais simples dos modelos escalares-tensoriais, o de Brans-Dicke, cujo espaço de fase estudaremos na seção 2.2.

\subsection{Modelo de Brans-Dicke}

O exemplo mais simples de STT é o chamado modelo de Brans-Dicke, em que a constante de gravitação de Newton é função do espaço e do tempo. Também se introduz um novo parâmetro, $\omega$, que permite recuperar os resultados da Relatividade Geral no limite $\omega \rightarrow \infty$. Uma motivação extra para alguns pesquisadores é o fato de que a criação do modelo de Brans-Dicke envolveu fundamentos teóricos como o Princípio de Mach e a hipótese dos grandes números de Dirac [53].

O interesse na teoria de Brans-Dicke como uma possível descrição da gravitação em escala cosmológica tem-se intensificado recentemente pela sua já referida relação com a teoria de cordas, e também por ser uma teoria capaz de ser testada no âmbito do Sistema Solar, através do mesmo tipo de experimentos efetuados para se obter os valores dos parâmetros pós-newtonianos (PPN), usados para se avaliar o nível de desvio que uma teoria de gravitação tem em relação à RG. Exemplos desse tipo de experimentos são o Lunar Laser Ranging e a sonda Cassini [44]. O primeiro deles, ao medir com grande precisão a distância Terra-Lua ao longo da várias décadas, vem confirmando cada vez mais a aplicabilidade da RG na escala do Sistema Solar, desfavorecendo o modelo de Brans-Dicke nessa região. A sonda Cassini, na sua passagem perto do Sol, enviou sinais de rádio que foram usados para mais uma vez confirmar a RG e definir que o valor mínimo do parâmetro de Brans-Dicke $\omega$ para que este modelo forneça valores coerentes no Sistema Solar deve ser maior que 40000.

Entretanto, é possivel argumentar [97] que a relação entre os modelos de gravidade modificada e os valores dos parâmetros PPN no Sistema Solar não é bem definida, estando baseada num limite que usualmente é tomado de maneira incorreta. Dessa forma, não se pode descartar o modelo de Brans-Dicke alegando que se trata de uma teoria de gravitação inválida em algumas escalas, e vamos considerar aqui a sua aplicação em escalas cosmológicas, em que sua validade é menos discutível. 
Neste modelo, escrevemos uma densidade Lagrangiana da forma [36]

$$
\mathcal{L}=\sqrt{-g}\left[-\Phi R+\frac{\omega}{\Phi} g^{\mu \nu} \partial_{\mu} \Phi \partial_{\nu} \Phi+\mathcal{L}_{m}\right]
$$

onde $\mathcal{L}_{m}$ é a densidade Lagrangiana para o campo de matéria, e as outras variáveis já foram definidas anteriormente.

As equações de Einstein se generalizam para

$$
G_{\mu \nu}=\frac{8 \pi}{\Phi} T_{\mu \nu}+\frac{\omega}{\Phi^{2}}\left(\Phi_{; \mu} \Phi_{; \nu}-\frac{1}{2} g_{\mu \nu} \Phi_{; \lambda}^{; \lambda}\right)+\frac{1}{\Phi}\left(\Phi_{; \mu \nu}-g_{\mu \nu} \square \Phi\right),
$$

sendo $T_{\mu \nu}$ o tensor de energia-momento para a matéria, exceto para o campo de BransDicke $\Phi$. A equação de movimento para $\Phi$ fica

$$
\square \Phi=\frac{8 \pi}{2 \omega+3} T
$$

onde $T=T_{\mu}^{\mu}$ é o traço do tensor de energia-momento.

Essas equações originam as equações cosmológicas a que aplicaremos a abordagem dos sistemas dinâmicos na próxima seção.

\subsection{Espaço de fase do modelo de Brans-Dicke}

Vamos iniciar aqui o estudo de um modelo de Brans-Dicke generalizado, em que incluimos um parâmetro $\omega$ não constante $(\omega=\omega(\phi))$ e um potencial do campo escalar $V(\phi)$ [54].

Em um espaço homogêneo e isotrópico, dotado da métrica de Friedmann-LemaîtreRobertson-Walker (FLRW)

$$
\mathrm{d} s^{2}=-\mathrm{d} t^{2}+a^{2}(t)\left[\frac{\mathrm{d} r^{2}}{1-K r^{2}}+r^{2}\left(\mathrm{~d} \theta^{2}+\sin ^{2} \theta \mathrm{d} \varphi^{2}\right)\right]
$$

onde $a$ é o fator de escala e $K$ é o índice de curvatura espacial, a gravitação pode ser descrita por uma ação do tipo

$$
S=\frac{1}{16 \pi} \int \mathrm{d}^{4} x \sqrt{-g}\left[\phi R-\frac{\omega(\phi)}{\phi} g^{a b} \nabla_{a} \phi \nabla_{b} \phi-V(\phi)\right]+S^{m}
$$

onde $S^{m}$ é a ação da matéria usual, $g$ é o determinante do tensor métrico. Nessa expressão, adotamos $G=1$ e índices latinos que variam de 0 a 3 . 
De (2.2.2), obtemos para as equações de campo:

$$
\begin{aligned}
& H^{2}=-H\left(\frac{\dot{\phi}}{\phi}\right)+\frac{\omega(\phi)}{6}\left(\frac{\dot{\phi}}{\phi}\right)^{2}+\frac{V(\phi)}{6 \phi}-\frac{K}{a^{2}}+\frac{8 \pi \rho^{m}}{3 \phi}, \\
& \dot{H}=-\frac{\omega(\phi)}{2}\left(\frac{\dot{\phi}}{\phi}\right)^{2}+2 H\left(\frac{\dot{\phi}}{\phi}\right)+\frac{1}{2(2 \omega+3) \phi}\left[\phi \frac{\mathrm{d} V}{\mathrm{~d} \phi}-2 V+\right. \\
& \left.+\frac{\mathrm{d} \omega}{\mathrm{d} \phi}(\dot{\phi})^{2}\right]+\frac{K}{a^{2}}-\frac{8 \pi}{(2 \omega+3) \phi}\left[(\omega+2) \rho^{m}+\omega P^{m}\right], \\
& \ddot{\phi}+\left(3 H+\frac{1}{2 \omega+3} \frac{\mathrm{d} \omega}{\mathrm{d} \phi}\right) \dot{\phi}=\frac{1}{2 \omega+3}\left[2 V-\phi \frac{\mathrm{d} V}{\mathrm{~d} \phi}+\right. \\
& \left.+8 \pi\left(\rho^{(m)}-3 P^{(m)}\right)\right] \text {, }
\end{aligned}
$$

onde $H \equiv \dot{a} / a$ é o parâmetro de Hubble e $\rho^{(m)}$ e $P^{(m)}$ são a densidade de energia e a pressão do fluido material, respectivamente.

A equação de estado do fluido material é parametrizada como

$$
P^{(m)}=w \rho^{(m)}
$$

sendo $w$ uma constante. Podemos ver, através da equação de conservação de energia

$$
\dot{\rho}^{(m)}+3 H\left(\rho^{(m)}+P^{(m)}\right)=0,
$$

que obtemos

$$
\rho^{(m)}=\frac{\rho_{0}}{a^{3(w+1)}},
$$

com $\rho_{0}$ constante.

As equações anteriores (2.2.3)-(2.2.5) constituem o sistema dinâmico cujo espaço de fase quadridimensional tem como variáveis $(a, \dot{a}, \phi, \dot{\phi})$.

Estamos prontos para escolher alguns tipos de funções de interesse teórico para exemplificar a análise da geometria do espaço de fase do modelo de Brans-Dicke, o que é feito nas próximas seções.

\subsubsection{O caso $V=\frac{1}{2} m^{2} \phi^{2}$ e $K=0$}

No trabalho anteriormente citado [54], o autor mostra o espaço de fase permitido para as órbitas das soluções dessas equações de campo em vários casos com diferentes potenciais 
e parâmetros $\omega$. Por exemplo, no caso do vácuo, espaço plano $(K=0)$ e potencial $V=\frac{1}{2} m^{2} \phi^{2}$, a equação $(2.2 .3)$ é rearranjada como (fazendo $m \equiv 1$ )

$$
\omega(\dot{\phi})^{2}-6 H \phi \dot{\phi}+\left(\frac{1}{2} \phi^{2}-6 H^{2} \phi\right) \phi=0
$$

que tem como soluções

$$
\dot{\phi}_{ \pm}(H, \phi)=\frac{1}{\omega}\left[3 H \phi \pm \sqrt{3(2 \omega+3) H^{2} \phi^{2}-\frac{1}{2} \omega \phi^{3}}\right] .
$$

Quando $K=0$, o fator de escala não aparece mais no lado direito das equações, a não ser através da constante de Hubble $H$. Assim, a dimensionalidade do espaço de fase fica reduzida às variáveis $(H, \phi, \dot{\phi})$ e podemos visualizar sua geometria de forma mais imediata, bem como trabalhar com um sistema dinâmico de equações mais simples.

Queremos analisar qualitativamente a geometria do espaço de fase, determinando em primeiro lugar as regiões que são proibidas às órbitas das soluções, ou seja, aquelas para as quais $\dot{\phi}$ tem valores reais. Adicionalmente, esperamos que essa análise nos permita inferir a forma das funções $\omega(\phi)$ e $V(\phi)$ que se ajustem melhor aos dados disponíveis sobre a estrutura do Universo.

O espaço de fase para esta situação é composto de uma superfície bidimensional com duas folhas, relacionadas aos sinais positivo e negativo na eq. (2.2.10). As Figuras 2.1-2.3 mostram o espaço de fase para a escolha $\omega=10$.

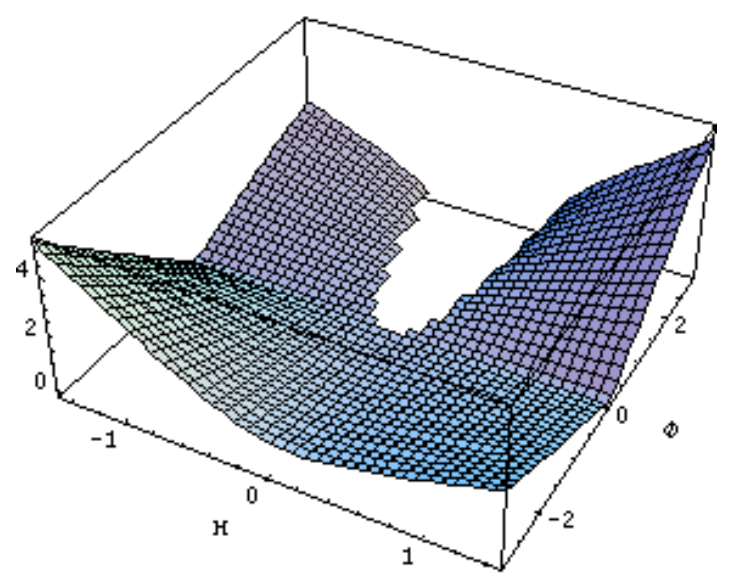

Figura 2.1: Folha superior do espaço de fase para um modelo com $\omega=10$ (Brans-Dicke), correspondendo ao sinal positivo na eq. (2.2.10) [54]. O "buraco" na superfície indica a região proibida às órbitas das soluções. No eixo vertical estão representados os valores para a superfícice de energia $\dot{\phi}$. 


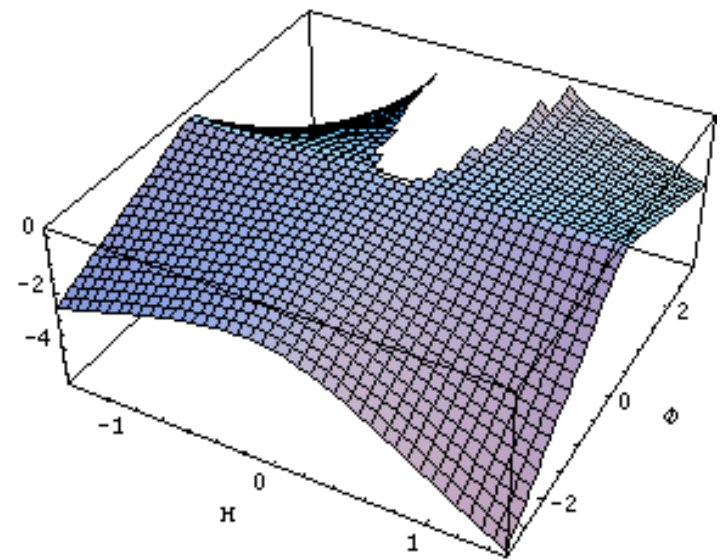

Figura 2.2: Folha inferior do espaço de fase, correspondendo agora ao sinal negativo na eq. (2.2.10), com $\dot{\phi}$ no eixo vertical.

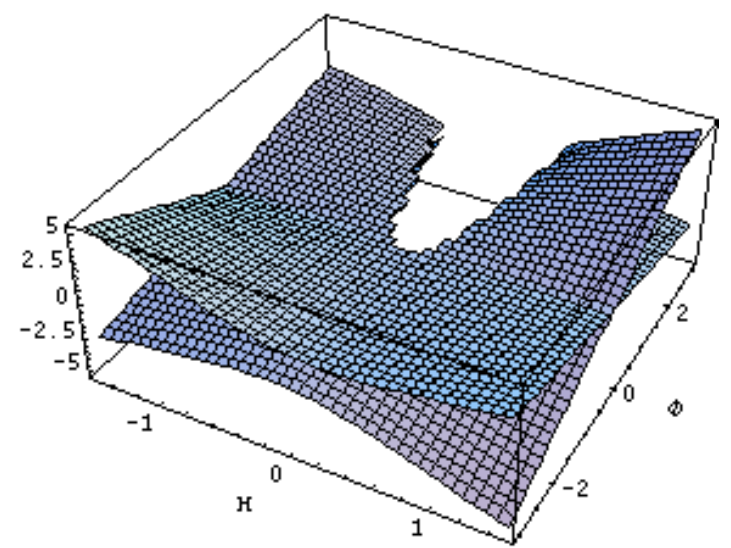

Figura 2.3: Espaço de fase para um modelo com $\omega=10$ (Brans-Dicke) [54], composto de duas folhas (correspondendo respectivamente aos sinais positivo e negativo na eq. (2.2.10)) ligadas pela borda da região proibida às órbitas das soluções. $\dot{\phi}$ é mostrado no eixo vertical.

Os pontos fixos para este sistema dinâmico, obtidos fazendo $\dot{H}=\dot{\phi}=0$, são soluções tipo de Sitter (que definem um determinado tipo de expansão para o Universo), dadas por

$$
H_{0}= \pm \sqrt{\frac{\phi_{0}}{12}}
$$

com $H_{0}$ e $\phi_{0}$ constantes. 


\subsubsection{O caso $V=\Lambda \phi$ e $K=0$}

Tendo como inspiração outros trabalhos [70, 71] que fornecem uma análise completa do espaço de fase para um modelo de Brans-Dicke com uma constante cosmológica $\Lambda$ (simplesmente fazendo $V(\phi)=\Lambda \phi$ na ação), podemos ilustrar a situação em que $\omega$ tem um valor muito mais alto e $w=-1$. Portanto, a densidade de energia do fluido é uma constante $\rho_{0}$. Deve-se enfatizar que recentes dados observacionais e de simulação numérica tendem a favorecer tal cenário ([72],[48]-[50]). As soluções neste caso são escritas como

$$
\dot{\phi}_{ \pm}(H, \phi)=\frac{1}{\omega}\left[3 H \phi \pm \sqrt{9 H^{2} \phi^{2}-\omega\left[\phi^{2}\left(\Lambda-6 H^{2}\right)+16 \pi \rho_{0}\right]}\right] .
$$

Com estas soluções, podemos mostrar o espaço de fase para uma escolha particular de constantes $\Lambda$, $\omega$ e $\rho_{0}$ (figuras 2.4-2.6).

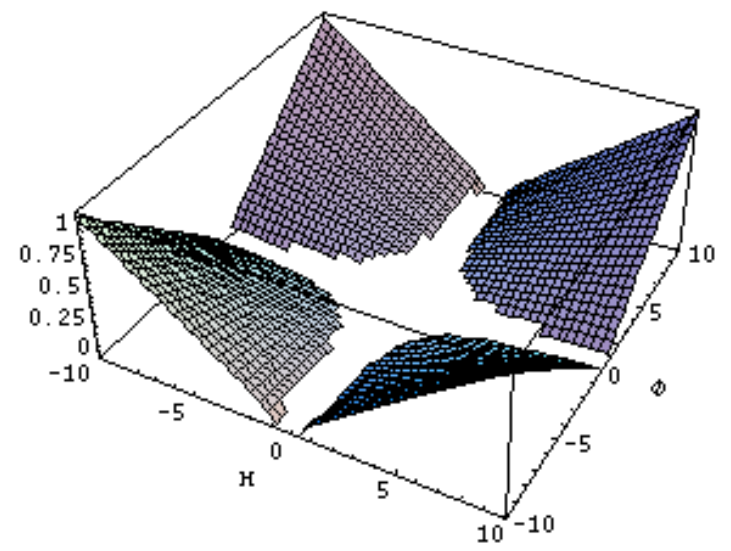

Figura 2.4: Folha superior do espaço de fase para um modelo de Brans-Dicke com uma constante cosmológica $\Lambda=1$, densidade de energia $\rho_{0}=2$ e parâmetro constante $\omega=50000$. $\dot{\phi}$ é mostrado no eixo vertical.

Passamos a procurar o sistema de equações dinâmicas para este modelo simples, como foi feito na seção anterior.

Chamando de $\Delta$ a expressão sob a raiz na eq.(2.2.12), podemos escrever a equação para $\dot{H}$ :

$$
\begin{aligned}
\dot{H}_{ \pm}= & -\frac{1}{2 \omega \phi^{2}}[3 H \phi \pm \sqrt{\Delta}]^{2}+\frac{2 H}{\omega}\left[3 H \pm \frac{\sqrt{\Delta}}{\phi}\right]- \\
& -\frac{1}{2(2 \omega+3)}\left(\frac{\Lambda}{2}+\frac{16 \pi \rho_{0}}{\phi}\right) .
\end{aligned}
$$




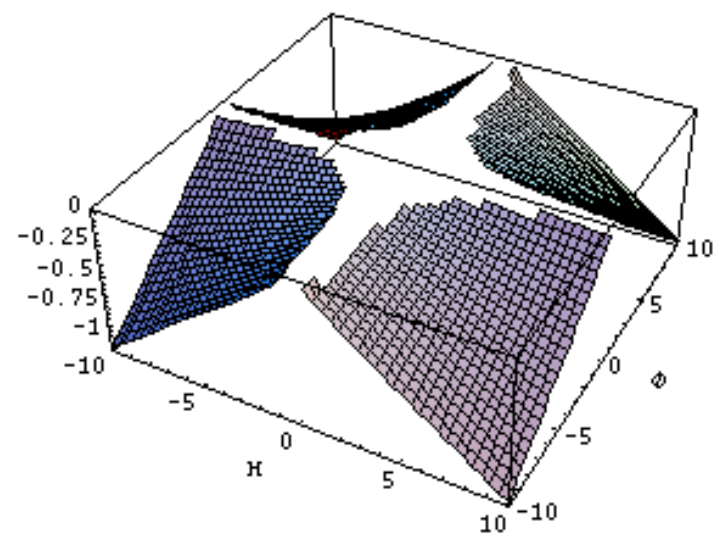

Figura 2.5: Folha inferior do espaço de fase, para o caso anterior.

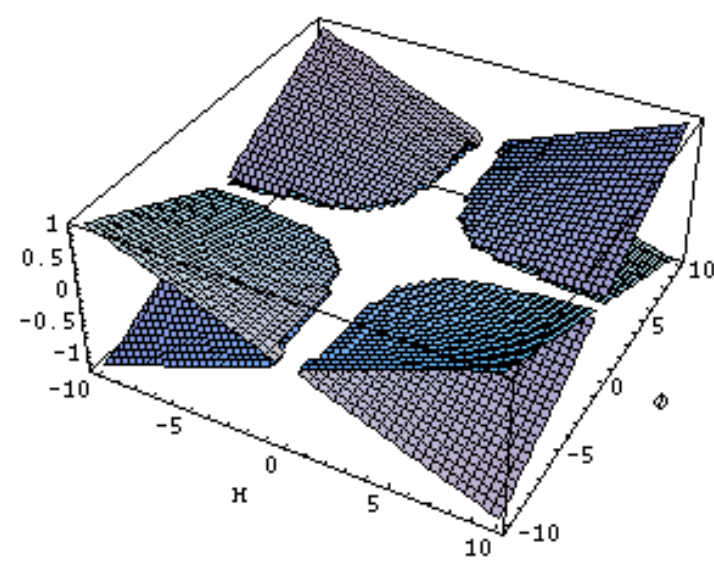

Figura 2.6: Espaço de fase completo.

Agora, as equações (2.2.12) e (2.2.13) formam o sistema que tem como pontos fixos

$$
H_{0}= \pm \sqrt{\frac{8 \pi \rho_{0}}{3 \phi_{0}^{2}}+\frac{\Lambda}{6}} .
$$

Essas são também soluções tipo de Sitter.

A estabilidade desses pontos fixos pode ser analisada por meio da função de Lyapunov $\mathcal{L}$ do sistema dinâmico (o chamado método direto [16]), com o objetivo de determinar, em ambos os casos mostrados aqui, se esses pontos fixos correspondem a soluções atratoras para uma grande quantidade de soluções representativas de uma variedade de condições iniciais, ou seja, pretende-se avaliar o tamanho da bacia de atração para essas soluções em relação aos pontos fixos do sistema.

Mostramos aqui um método de análise de modelos escalares-tensoriais usados como 
uma alternativa à gravitação de Einstein. Vimos que foi necessário incluir um campo escalar extra cuja dinâmica em princípio é responsável pela aceleração atual do Universo. Obviamente, essa não é única teoria de gravitação modificada possível e, no próximo capítulo, apresentaremos uma análise similar para uma classe de modelos que faz uso de uma abordagem diferente para implementar a generalização da gravitação. Como esses modelos envolvem uma mudança na ação de Einstein-Hilbert com a inclusão de uma função do escalar de curvatura $R$ (caracterizando-se, assim, por uma alteração na propria geometria do espaço-tempo), eles são chamados de modelos $f(R)$.

Mostraremos que esses dois enfoques para a gravitação não são completamente independentes, existindo um relação de equivalência entre eles, pelo menos em alguns casos particulares. 


\section{Capítulo 3}

\section{Modelos de gravidade $f(R)$}

Uma classe de modelos que tem sido muito investigada nos últimos anos é o de gravidade $f(R)$, em que se consideram modificações da própria geometria do espaço-tempo ${ }^{1}$. A ação é escrita agora como

$$
S=\frac{1}{16 \pi G} \int \mathrm{d}^{4} x \sqrt{-g} f(R)+S_{m}\left[g_{\mu \nu}, \psi_{m}\right]
$$

onde $S_{m}$ é a ação de matéria.

Aqui estudamos estes modelos no chamado formalismo métrico, em que se realiza a variação da ação em relação à métrica. ${ }^{2}$ Neste formalismo, as equações de campo se escrevem (após adotarmos $8 \pi G=1$ )

$$
f^{\prime}(R) R_{a b}-\frac{f(R)}{2} g_{a b}=\nabla_{a} \nabla_{b} f^{\prime}-g_{a b} \square f^{\prime}+T_{a b}
$$

Com o intuito de estudar o espaço de fase desses modelos o mais genericamente possível, consideramos um Universo homogêneo e isotrópico, dotado da métrica de Friedmann-Lemaître-Robertson-Walker, com curvatura espacial nula,

$$
d s^{2}=-d t^{2}+a^{2}(t) d \mathbf{x}^{2}
$$

onde $a(t)$ é o fator de escala do Universo. Neste cenário, as equações de campo se reduzem a

$$
H^{2}=\frac{1}{3 f^{\prime}}\left[\frac{R f^{\prime}-f}{2}-3 H \dot{R} f^{\prime \prime}\right]
$$

\footnotetext{
${ }^{1}$ Este capítulo é baseado nos resultados apresentados em [111].

${ }^{2}$ Há outras formas de se efetuar esta variação, o que dá origem aos formalismos de Palatini e métricoafim [106, 107]. Esses casos não serão tratados aqui.
} 


$$
2 \dot{H}+3 H^{2}=-\frac{1}{f^{\prime}}\left[\dot{R}^{2} f^{\prime \prime \prime}+2 H \dot{R} f^{\prime \prime}+\ddot{R} f^{\prime \prime}+\frac{1}{2}\left(f-R f^{\prime}\right)\right],
$$

onde a matéria foi ignorada, já que estamos interessados num regime em que a aceleração do Universo é dominada pela gravidade modificada. Nas expressões anteriores, uma aspa significa derivada em relação a $R$ e um ponto, derivada temporal.

Lembrando que definimos $H=\dot{a} / a$, temos um sistema dinâmico que se reduz às variáveis $(H, R, \dot{R})$, e que é representado pela superfície de energia $\Sigma$ determinada por

$$
\dot{R}=\frac{R f^{\prime}-f-6 f^{\prime} H^{2}}{6 H f^{\prime \prime}} .
$$

Assumimos que $f^{\prime}>0$, para que tenhamos um acoplamento gravitacional positivo, e também excluímos a possibilidade $f^{\prime \prime}<0$, que leva a instabilidades na teoria [108, 110].

Notamos que o espaço de fase tem agora apenas uma folha, resultado do fato de que para cada valor do par $(H, R)$ há apenas um valor possível para $\dot{R}$. Esta situação é diferente do que se observa para os modelos escalares-tensoriais, em que, em geral, têmse dois valores para a variável $\dot{\phi}(H, \phi)$, devido ao fato de que o vínculo hamiltoniano (3.0.4) é quadrático em $\phi$ (ver equação (2.2.12) e figura 2.6).

A região dinamicamente proibida, correspondente a $H^{2}<0$, é dada pela união $\mathcal{F}=\mathcal{F}_{1} \cup \mathcal{F}_{2}$, onde

$$
\mathcal{F}_{1} \equiv\left\{(H, R): R f^{\prime}-f-6 H \dot{R} f^{\prime \prime}<0\right\}
$$

$\mathrm{e}$

$$
\mathcal{F}_{2} \equiv\left\{(H, R): f, f^{\prime} \text { ou } f^{\prime \prime} \text { não são definidos }\right\}
$$

A borda do subconjunto conexo $\mathcal{F}_{1}$ da região proibida é

$$
\mathcal{B} \equiv\left\{(H, R, \dot{R}): f^{\prime} H^{2}=0\right\}
$$

que define uma curva na superfície $\Sigma$ ao longo da qual a densidade de energia efetiva $\rho_{\text {eff }}$ (ver eq. (3.1.1)) se anula.

O fato de haver uma superfície com apenas uma folha impede a existência de dinâmica caótica para esses modelos ${ }^{3}$.

\footnotetext{
${ }^{3} \mathrm{O}$ famoso teorema de Poincaré-Bendixson não se aplica aqui, já que o espaço de fase não corresponde a uma superfície plana, compacta e simplesmente conexa. Entretanto, a ausência de caos já foi demonstrada [93] para casos gerais de modelos escalares-tensoriais, cujo espaço de fase apresenta duas folhas, e a prova é facilmente extensível ao caso mais simples dos espaços de fase compostos por apenas uma folha dos modelos $f(R)$.
} 
A fim de exemplificar a geometria do espaço de fase que foi investigado de forma genérica até agora, usamos duas formas da função $f(R)$ para construir as figuras 3.1 e 3.2.

i. $f(R)=R+\alpha R^{2}$

Essa função é usada para descrever a fase inflacionária do Universo.

Neste caso, a superfície de energia é expressa por

$$
\dot{R}=\frac{\alpha R^{2}-12 \alpha R H^{2}-6 H^{2}}{12 \alpha H} .
$$

Para essa função, a região dinamicamente proibida é

$$
\mathcal{F}=\left\{(H, R): 6+12 \alpha R H^{2}<0\right\} .
$$

Notamos que não há pontos na região proibida enquanto mantivermos $R>0$ (correspondente a um Universo em expansão).

Escolhendo $\alpha=0.0001$, o espaço de fase é esquematizado na figura 3.1.

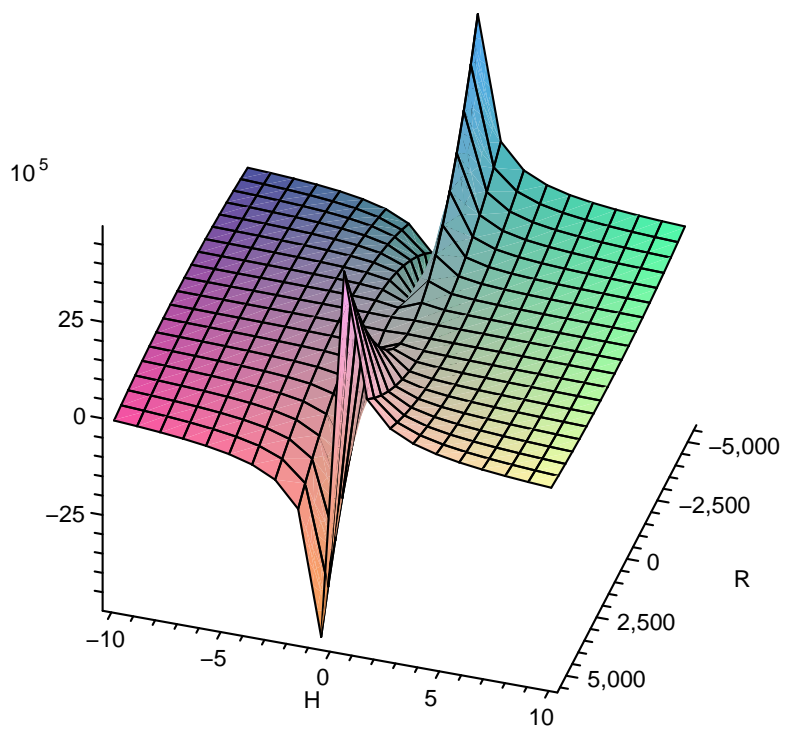

Figura 3.1: Espaço de fase para o modelo de gravidade modificada com $f(R)=R+\alpha R^{2}$, com $\alpha=0.0001$. Notamos que há apenas uma folha para a superfície $\dot{R}$ (que tem valores representados no eixo vertical).

ii. $f(R)=R-\frac{\mu^{4}}{R}$ 
A superfície de energia é dada por

$$
\dot{R}=\frac{\mu^{4} R+\mu^{4} R^{2}-6 H^{2}\left(R^{3}+\mu^{4} R\right)}{-12 H \mu^{4}} .
$$

A região dinamicamente proibida é agora

$$
\mathcal{F}=\left\{(H, R): 6 H^{2}\left(1+\frac{\mu^{4}}{R^{2}}\right)<0\right\}
$$

Os únicos valores proibidos são aqueles para os quais $R=0$.

Escolhendo $\mu^{4}=0.0001$, o espaço de fase pode ser desenhado como na figura 3.2.

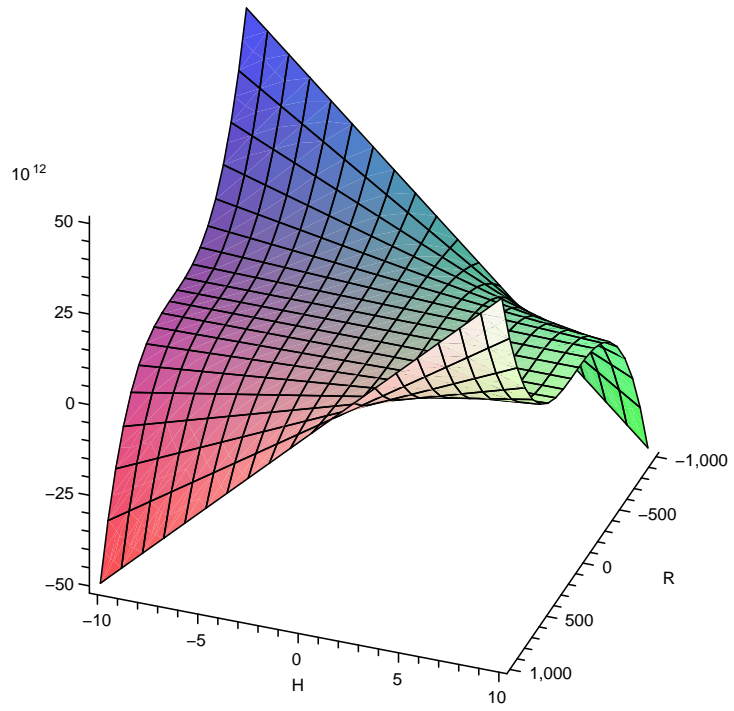

Figura 3.2: Espaço de fase para o modelo de gravidade modificada com $f(R)=R-\frac{\mu^{4}}{R}$, com $\mu^{4}=$ 0.0001. $\dot{R}$ é mostrado no eixo vertical.

É preciso notar aqui, ao contrário do que pode ocorrer no caso dos modelos escalarestensoriais, a superfície de energia não apresenta "buracos", devido ao fato de que a região proibida não a intercepta.

Os pontos fixos do sistema, para os quais $(\dot{H}, \dot{R}) \equiv 0$, são espaços de de Sitter

$$
(H, R, \dot{R})=\left(H_{0}, 12 H_{0}^{2}, 0\right)=\left( \pm \sqrt{\frac{f_{0}}{6 f_{0}^{\prime}}}, \frac{2 f_{0}}{f_{0}^{\prime}}, 0\right)
$$

onde $f_{0} \equiv f\left(R_{0}\right), f_{0}^{\prime} \equiv f^{\prime}\left(R_{0}\right)$, etc. Estes pontos fixos existem apenas com a condição de que $f_{0} / f_{0}^{\prime} \geqslant 0$. 
Dadas as condições $f_{0} \geqslant 0$ e $f_{0}^{\prime}>0$, dois pontos de equilíbrio de de Sitter sempre existem no plano $\dot{R}=0$, sendo um que se refere à contração e o outro à expansão, com valores $H_{0}^{( \pm)}= \pm \sqrt{\frac{f_{0}}{6 f_{0}^{\prime}}}$. No caso em que $f\left(R_{0}\right)=0$, esses pontos degeneram em um único, o espaço de Minkowski, que se situa na fronteira da região proibida.

Os pontos fixos de de Sitter mostram estabilidade em relação a perturbações homogêneas e inomogêneas [55] com a condição

$$
\frac{\left(f_{0}^{\prime}\right)^{2}-2 f_{0} f_{0}^{\prime \prime}}{f_{0}^{\prime} f_{0}^{\prime \prime}} \geqslant 0 .
$$

Os espaços de de Sitter em contração, com $H_{0}<0$, são sempre instáveis [91], e, em ordem linear, aqueles em expansão são sempre estáveis em relação aos modos tensoriais. Os pontos de equilíbrio $(H, R, \dot{R})=\left(H_{0}, 12 H_{0}^{2}, 0\right)$ sempre se situam no plano $(H, R)$ do espaço de fase.

A fim de permitir o estudo de simetrias pontuais nos modelos $f(R)$, é útil escrever a Lagrangiana $L$ e a Hamiltoniana $E$ efetivas para estas teorias, num espaço de FLRW e escolhendo $a$ e $R$ como coordenadas Lagrangianas.

Podemos escrever a Lagrangiana efetiva para os modelos $f(R)$ como

$$
L(a, R, \dot{a}, \dot{R})=a^{3}\left[6 H^{2} f^{\prime}+6 H f^{\prime \prime} \dot{R}+f^{\prime} R-f\right]
$$

Os momentos canonicamente conjugados às coordenadas generalizadas $a$ e $R$ são

$$
p_{a}=\frac{\partial L}{\partial \dot{a}}=6 a^{2}\left(2 H f^{\prime}+f^{\prime \prime} \dot{R}\right), \quad p_{R}=\frac{\partial L}{\partial \dot{R}}=6 a^{3} H f^{\prime \prime},
$$

e a Hamiltoniana efetiva (que também pode ser chamada de energia efetiva) resulta em

$$
E(a, R, \dot{a}, \dot{R})=p_{a} \dot{a}+p_{R} \dot{R}-L=a^{3}\left(6 H^{2} f^{\prime}+6 H f^{\prime \prime} \dot{R}-f^{\prime} R+f\right) .
$$

O vínculo hamiltoniano (3.0.4) corresponde a $E=0$.

Através da equação de Euler-Lagrange

$$
\frac{d}{d t}\left(\frac{\partial L}{\partial \dot{R}}\right)-\frac{\partial L}{\partial R}=0
$$

obtemos a conhecida relação

$$
R=6\left(\frac{\ddot{a}}{a}+\frac{\dot{a}^{2}}{a^{2}}\right)
$$

enquanto a equação

$$
\frac{d}{d t}\left(\frac{\partial L}{\partial \dot{a}}\right)-\frac{\partial L}{\partial a}=0
$$


fornece a equação (3.0.5).

Por meio da aplicação do teorema de Noether, a Lagrangiana e a Hamiltoniana obtidas anteriormente podem levar à reconstrução da função $f(R)$, de forma similar a estudos feitos para teorias escalares-tensoriais [112].

Para Universos dotados de métrica FLRW espacialmente não-planos $(k \neq 0)$, o vínculo hamiltoniano tem a forma

$$
H^{2}=\frac{1}{3 f^{\prime}}\left[\frac{R f^{\prime}-f}{2}-3 H \dot{R} f^{\prime \prime}\right]-\frac{k}{a^{2}},
$$

onde o fator de escala $a$ aparece explicitamente, impossibilitando o uso de $H$ em vez de $a$ como variável. O caso estudado aqui, através da superfície de energia $\Sigma$ para $k=0$, separa o caso $k<0$ de $k>0$, impedindo que as órbitas cruzem a superfície $\Sigma$. No caso $k=0$, as órbitas ficam compreendidas totalmente em $\Sigma$.

\subsection{A equação de estado efetiva}

É interessante tentar definir quantidades que possam servir como parâmetros observacionais dos modelos $f(R)$. Uma dessas quantidades, particularmente relevante, é o parâmetro de equação de estado $w$. Veremos aqui como ele pode ser definido para os modelos que estamos investigando.

Usando as eqs. (3.0.4) e (3.0.5) e identificando o acoplamento gravitacional com $f^{\prime}(R)$, pode-se escrever a densidade de energia e a pressão efetivas para os modelos $f(R)$ da seguinte forma:

$$
\begin{gathered}
\rho_{e f f}=\frac{R f^{\prime}-f}{2}-3 H \dot{R} f^{\prime \prime}, \\
P_{e f f}=\dot{R}^{2} f^{\prime \prime \prime}+2 H \dot{R} f^{\prime \prime}+\ddot{R} f^{\prime \prime}+\frac{1}{2}\left(f-R f^{\prime}\right) .
\end{gathered}
$$

A densidade de energia efetiva $\rho_{\text {eff }}$ é não-negativa, como se pode ver por inspeção da eq. (3.0.4). O parâmetro de equação de estado efetivo $w_{\text {eff }}$ pode ser expresso como

$$
w_{e f f} \equiv \frac{P_{e f f}}{\rho_{e f f}}=\frac{\dot{R^{2}} f^{\prime \prime \prime}+2 H \dot{R} f^{\prime \prime}+\ddot{R} f^{\prime \prime}+\frac{1}{2}\left(f-R f^{\prime}\right)}{\frac{R f^{\prime}-f}{2}-3 H \dot{R} f^{\prime \prime}} .
$$

A equação (3.0.4) garante que o denominador no lado direito da eq. (3.1.3) é estritamente positivo, assim, o sinal da equação de estado efetiva é determinado pelo numerador. 
Medidas recentes, resultantes de observações de supernovas tipo Ia, de radiação cósmica de fundo e de redshift de galáxias, embora ainda não plenamente conclusivas, favorecem uma equação de estado efetiva $w \approx-1$ no presente. Para que o modelo reproduza a equação de estado que corresponde a uma expansão do tipo de Sitter com $w_{\text {eff }}=-1$, ele deve ter

$$
\frac{f^{\prime \prime \prime}}{f^{\prime \prime}}=\frac{\dot{R} H-\ddot{R}}{(\dot{R})^{2}} .
$$

É conveniente introduzir a quantidade $\psi(R) \equiv f^{\prime}(R)$ para escrever

$$
w_{e f f}=-1+2 \frac{(\ddot{\psi}-H \dot{\psi})}{R \psi-f-6 H \dot{\psi}}=-1+\frac{(\ddot{\psi}-H \dot{\psi})}{3 \psi H^{2}} .
$$

Alternativamente, o desvio da equação de estado de de Sitter $w=-1$ pode ser parametrizado por

$$
\rho_{e f f}+P_{e f f}=\frac{\ddot{\psi}-H \dot{\psi}}{\psi}=\frac{\dot{\psi}}{\psi} \frac{d}{d t}\left[\ln \left(\frac{\dot{\psi}}{a}\right)\right] .
$$

De acordo com eq. (3.1.6), uma solução de de Sitter exata corresponde a $\dot{\psi}=f^{\prime \prime}(R) \dot{R}=$ 0 , ou a $\dot{\psi}=C a(t)=C a_{0} \mathrm{e}^{H_{0} t}$, onde $C \neq 0$ é uma constante de integração. É fácil ver que a segunda solução para $\psi(t)$ não é aceitável porque leva à equação absurda $f^{\prime \prime}(R) \dot{R}=C a_{0} \mathrm{e}^{H_{0} t}$ em que o lado esquerdo é independente do tempo (para uma solução de de Sitter) enquanto o lado direito não é.

A equação (3.1.4) pode ser útil para restringir as possíveis funções $f(R)$ que sejam cosmologicamente relevantes e o parâmetro $w_{\text {eff }}$, como dado pela equação (3.1.5), poderá ser comparado com resultados de futuras determinações experimentais que serão efetuadas nos próximos anos.

\subsection{A teoria escalar-tensorial equivalente}

Uma ação gravitacional modificada da forma (3.0.1) pode ser reescrita como uma teoria escalar-tensorial equivalente $[102,103,104]$. Quando $f^{\prime \prime} \neq 0$, a ação de gravidade modificada pode ser reescrita como a teoria escalar-tensorial

$$
S=\frac{1}{2} \int d^{4} x \sqrt{-g}[\psi(\phi) R-V(\phi)]+S^{(m)},
$$

onde

$$
\psi(\phi)=f^{\prime}(\phi), \quad V(\phi)=\phi f^{\prime}-f, \quad \phi=R
$$


e o parâmetro de Brans-Dicke é $\omega=0$ [102, 103, 104]. Identificamos o escalar de curvatura $R$ com o campo escalar $\phi$, e o mapeamento em variáveis escalares-tensoriais pode ser visto como uma transformação de Legendre. De fato, a variação da ação 3.2.1 em relação a $\phi$ resulta em

$$
R \frac{d \psi}{d \phi}-\frac{d V}{d \phi}=0
$$

que por sua vez resulta em $\phi=R$ se $f^{\prime \prime} \neq 0$. Não há um termo cinético para o escalar $\phi$ na ação (3.2.1), mas esta quantidade é dinâmica, já que obedece à equação dinâmica (3.0.2).

Na métrica (2.2.1) as equações de campo se tornam

$$
\begin{gathered}
H^{2}=\frac{1}{6 \psi}\left[V(\phi)-6 H \psi^{\prime} \dot{\phi}\right], \\
\dot{H}=\frac{1}{2 \psi}\left(H \psi^{\prime} \dot{\phi}-\psi^{\prime} \ddot{\phi}-\psi^{\prime \prime} \dot{\phi}^{2}\right),
\end{gathered}
$$

e (3.2.3), ou

$$
\begin{gathered}
H^{2}=\frac{1}{6 f^{\prime}}\left(\phi f^{\prime}-f-6 H f^{\prime \prime \prime} \dot{\phi}\right), \\
\dot{H}=\frac{1}{2 f^{\prime}}\left(H f^{\prime \prime} \dot{\phi}-f^{\prime \prime} \ddot{\phi}-f^{\prime \prime \prime} \dot{\phi^{2}}\right), \\
(R-\phi) f^{\prime \prime}=0 .
\end{gathered}
$$

Encarando o vínculo hamiltoniano (3.2.4) como uma equação algébrica para $\dot{\phi}$, pode-se eliminar esta variável do espaço de fase tridimensional $(H, \phi, \dot{\phi})$ (que corresponde ao espaço de fase $(H, R, \dot{R}))$, obtendo-se

$$
\dot{\phi}(H, \phi)=\frac{1}{6 H f^{\prime \prime}}\left(\phi f^{\prime}-f-6 f^{\prime} H^{2}\right) .
$$

Esta equação representa uma superfície de energia com apenas uma folha, que corresponde ao caso especial $\omega=0$ da cosmologia escalar-tensorial [54], como vimos na seção anterior. Os pontos de equilíbrio $(\dot{H}, \dot{\phi})=(0,0)$ são espaços de de Sitter com campo escalar constante e satisfazem

$$
\left(H_{0}, \phi_{0}\right)=\left( \pm \sqrt{\frac{f_{0}}{6 f_{0}^{\prime}}}, \frac{2 f_{0}}{f_{0}^{\prime}}\right) .
$$

Em teorias gerais de gravidade escalar-tensorial pode-se ter soluções de de Sitter com campo escalar não constante (as quais, entretanto, não são pontos fixos). No equivalente escalar-tensorial da gravidade $f(R)$ isto não é possível por causa do vínculo $\phi=R=$ 
$12 H_{0}^{2}$ que força $\phi$ a ser constante para todas as soluções de de Sitter, que têm parâmetro de Hubble $H_{0}$ constante.

A condição de estabilidade invariante de gauge para estes pontos de equilíbrio de de Sitter em relação a perturbações inomogêneas, obtida em [55, 95], é novamente

$$
\frac{\left(f_{0}^{\prime}\right)^{2}-2 f_{0} f_{0}^{\prime \prime}}{f_{0}^{\prime} f_{0}^{\prime \prime}} \geqslant 0 .
$$

A Lagrangiana e a Hamiltoniana efetivas para a teoria escalar-tensorial (3.2.1) são agora dadas por

$$
L(a, \phi, \dot{a}, \dot{\phi})=a^{3}\left(6 H^{2} \psi+6 H \dot{\psi}+V\right)
$$

e

$$
E(a, \phi, \dot{a}, \dot{\phi})=a^{3}\left(6 H^{2} \psi+6 H \dot{\psi}-V\right) .
$$

Estas podem ser obtidas a partir de Lagrangianas bem conhecidas de teorias escalarestensoriais ([11] e referências internas). 


\section{Capítulo 4}

\section{Singularidades em modelos $f(R)$ anisotrópicos}

Até aqui, estudamos alguns modelos escalares-tensoriais e $f(R)$ em espaços-tempos homogêneos e isotrópicos definidos pela métrica de FLRW. Entretanto, é possível investigar esses modelos também em uma gama de espaços-tempos anisotrópicos, analisando interessantes aspectos que possam surgir. Por exemplo, os modelos de Bianchi constituem um conjunto de métricas anisotrópicas que podem ser utilizadas para implementar o estudo do efeito das anisotropias em modelos cosmológicos [11].

Faremos neste capítulo uma breve revisão de estudos recentes [115, 116] sobre a aplicação do Princípio de Maupertuis-Jacobi a cosmologias que envolvem um campo escalar não-minimamente acoplado à curvatura na ação gravitacional. Este princípio identifica as equações de movimento de um sistema mecânico com as geodésicas desse sistema em uma variedade pseudo-Riemanniana dotada de uma métrica construída a partir da Lagrangiana original. As condições para a existência dessa métrica levam ao reconhecimento de singularidades do modelo.

Em seguida, aplicaremos este método ao estudo do modelo $f(R)$, tanto no caso de uma métrica de FRLW como no de uma métrica anisotrópica do tipo mais simples, conhecida como Bianchi tipo I. Estamos interessados na ocorrência de singularidades neste tipo de modelo e na possibilidade das mesmas serem obtidas de uma forma mais direta (através de argumentos geométricos) do que se verifica com a análise dinâmica. 


\subsection{O Princípio de Maupertuis-Jacobi em modelos de acoplamento não-mínimo}

Em mecânica clássica, o Princípio de Maupertuis-Jacobi (em sua formulação moderna) estabelece que a dinâmica de um sistema pode ser representada por movimentos geodésicos em uma variedade Riemanniana associada. Descrevendo o sistema clássico com $N$ graus de liberdade por meio da Lagrangiana

$$
L(q, \dot{q})=\frac{1}{2} g_{i j}(q) \dot{q}^{i} \dot{q}^{j}-V(q),
$$

onde $i, j=1,2, \ldots, N$, um ponto representa derivada temporal e $g_{i j}$ é a métrica de Riemann da variedade $N$-dimensional $\mathcal{M}$, as equações de movimento se escrevem

$$
\ddot{q}^{i}+\Gamma_{j k}^{i} \dot{q}^{j} \dot{q}^{k}=-g^{i j} \partial_{j} V(q)
$$

A Hamiltoniana do sistema descrito por (4.1.1) é

$$
\mathcal{H}(q, p)=\frac{1}{2} g^{i j}(q) p_{i} p_{j}+V(q)
$$

onde $p_{i}=g_{i j} \dot{q}^{j}$, e representa a energia total, sendo portanto uma constante de movimento.

A região permitida às órbitas das soluções no espaço de configurações é dada por

$$
\mathcal{D}_{E}=\{q \in \mathcal{M}: V(q) \leq E\}
$$

O princípio de Maupertuis-Jacobi estabelece que as equações de movimento (4.1.2), no interior de $\mathcal{D}_{E}$, são equivalentes à equação geodésica da geometria de Riemann em $\mathcal{M}$ definida pela métrica de Jacobi

$$
\hat{g}_{i j}(q)=2(E-V(q)) g_{i j}(q)
$$

A equação geodésica associada é dada por

$$
\hat{\nabla}_{u} u=\frac{d^{2} q^{i}}{d s^{2}}+\hat{\Gamma}_{j k}^{i} \frac{d q^{j}}{d s} \frac{d q^{k}}{d s}=0
$$

onde $u=d q^{i} / d s$ é o vetor tangente ao longo da geodésica, $\hat{\nabla}$ e $\hat{\Gamma}_{j k}^{i}$ são, respectivamente, a derivada covariante e a conexão de Levi-Civita para a métrica de Jacobi $\hat{g}_{i j}$ e $s$ é um parâmetro ao longo da geodésica obedecendo a

$$
\frac{d s}{d t}=2(E-V(q))
$$


O princípio foi utilizado recentemente em [114] no estudo de modelos cosmológicos homogêneos e isotrópicos com $N$ campos minimamente acoplados $\phi^{\alpha}$ autointeragentes com valores num espaço de Riemann dotado de uma métrica $G_{\alpha \beta}$, e com ação

$$
S=\int d^{4} x \sqrt{-g}\left\{R-g^{i j} G_{\alpha \beta}(\phi) \partial_{i} \phi^{\alpha} \partial_{j} \phi^{\beta}-2 V(\phi)\right\} .
$$

Usando a métrica de FLRW, os autores mostraram que as equações de movimento, deduzidas a partir da ação (4.1.8), correspondem à geodésica de uma certa métrica efetiva de Jacobi em uma variedade Lorentziana. Eles usaram esse formalismo para identificar fases assintóticas de expansão acelerada em modelos com potencial exponencial.

É possível incluir nessa análise os modelos de campo escalar não-minimamente acoplados, generalizando a ação (4.1.8) da forma

$$
S=\int d^{4} x \sqrt{-g}\left\{F(\phi) R-g^{i j} G_{\alpha \beta}(\phi) \partial_{i} \phi^{\alpha} \partial_{j} \phi^{\beta}-2 V(\phi)\right\},
$$

lembrando que estamos considerando um espaço-tempo de FLRW com parâmetro de curvatura $k=0$ e definindo $H=\dot{a} / a$. A partir dessa ação obtém-se a Lagrangiana

$$
L\left(a, \dot{a}, \phi_{\alpha}, \dot{\phi}^{\alpha}\right)=a^{3}\left(-6 H^{2} F-6 H \dot{\phi}^{\alpha} \partial_{\alpha} F+G_{\alpha \beta}(\phi) \dot{\phi}^{\alpha} \dot{\phi}^{\beta}-2 V(\phi)\right) .
$$

Introduzindo a métrica Lorentziana

$$
G_{A B}\left(a, \phi^{\alpha}\right)=\left(\begin{array}{cc}
-6 a F & -3 a^{2} \partial_{\beta} F \\
-3 a^{2} \partial_{\alpha} F & a^{3} G_{\alpha \beta}
\end{array}\right),
$$

no espaço de configurações, com índices romanos maiúsculos variando de 1 até $N+1$, a Lagrangiana (4.1.10) pode ser escrita na forma

$$
L\left(\phi_{A}, \dot{\phi}^{A}\right)=G_{A B}(\phi) \dot{\phi}^{A} \dot{\phi}^{B}-2 V_{e f f}\left(\phi^{A}\right),
$$

onde $\phi^{A}=\left(a, \phi^{\alpha}\right)$ e $V_{\text {eff }}\left(\phi^{A}\right)=a^{3} V\left(\phi^{\alpha}\right)$. Um ponto importante a se ressaltar é que, para que a quantidade $G_{A B}$ represente verdadeiramente uma métrica, seu determinante deve ser não-nulo.

A Hamiltoniana associada a (4.1.10) é

$$
\mathcal{H}\left(\phi^{A}, \pi_{A}\right)=G^{A B} \pi_{A} \pi_{B}+2 V_{\text {eff }}\left(\phi^{A}\right),
$$

onde $\pi_{A}=G_{A B} \dot{\phi}^{B}$. Esta função é uma constante de movimento e o vínculo de energia implica $\mathcal{H}=0$. 
A imposição det $G_{A B} \neq 0$ está relacionada com a ausência de algumas singularidades dinâmicas no modelo. Por exemplo, para o caso $N=1$ e $G_{\alpha \beta}=1$, temos

$$
\operatorname{det} G_{A B}=-6 a^{4}\left(F(\phi)+\frac{3}{2}\left(F^{\prime}(\phi)\right)^{2}\right)
$$

Assim, a situação em que $\operatorname{det} G_{A B}=0$ determina uma singularidade que envolve o campo escalar da forma

$$
F(\phi)+\frac{3}{2}\left(F^{\prime}(\phi)\right)^{2}=0
$$

Vale enfatizar que esta singularidade é agora geométrica e não mais apenas dinâmica. Ela é bem conhecida no contexto dos modelos escalares-tensoriais [11, 32], e não é surpresa a sua presença aqui.

É interessante notar que o acoplamento conforme, para o qual $F(\phi)=1-\phi^{2} / 6$, pode evitar naturalmente a singularidade definida por $\operatorname{det} G_{A B}=0$, dado que, para este tipo de acoplamento,

$$
F(\phi)+\frac{3}{2}\left(F^{\prime}(\phi)\right)^{2}=1
$$

\subsection{Métrica anisotrópica}

Vamos agora aplicar o mesmo método a uma métrica anisotrópica $D$-dimensional de Bianchi tipo I

$$
d s^{2}=-d t^{2}+\sum_{i=1}^{D-1} a_{i}^{2}(t) d x^{i}
$$

que implica para o escalar de curvatura

$$
R=2\left(\sum_{i=1}^{D-1}\left(\dot{H}_{i}+H^{2}\right)+\sum_{i=1, j>i}^{D-1} H_{i} H_{j}\right)
$$

onde $H_{i}=\dot{a}_{i} / a_{i}$.

Da ação (4.1.9), obtemos a Lagrangiana

$$
\begin{aligned}
L= & \left(\prod_{i=1}^{D-1} a_{i}\right)\left(-2 F(\phi) \sum_{i=1, j>i}^{D-1} H_{i} H_{j}-2\left(\sum_{i=1}^{D-1} H_{i}\right) \dot{\phi}^{\alpha} \partial_{\alpha} F(\phi)+\right. \\
& \left.+G_{\alpha \beta}(\phi) \dot{\phi}^{\alpha} \dot{\phi}^{\beta}-2 V(\phi)\right) .
\end{aligned}
$$


No caso $D=4$, introduzimos a métrica Lorentziana

$$
G_{A B}=\left(\begin{array}{cccc}
0 & -a_{3} F & -a_{2} F & -a_{2} a_{3} \partial_{\beta} F \\
-a_{3} F & 0 & -a_{1} F & -a_{1} a_{3} \partial_{\beta} F \\
-a_{2} F & -a_{1} F & 0 & -a_{1} a_{2} \partial_{\beta} F \\
-a_{2} a_{3} \partial_{\alpha} F & -a_{1} a_{3} \partial_{\alpha} F & -a_{1} a_{2} \partial_{\alpha} F & a_{1} a_{2} a_{3} G_{\alpha \beta}
\end{array}\right) .
$$

Aqui, vale $\phi^{A}=\left(a_{1}, a_{2}, a_{3}, \phi^{\alpha}\right) \mathrm{e}$

$$
V_{e f f}\left(\phi^{A}\right)=a_{1} a_{2} a_{3} V\left(\phi^{\alpha}\right)
$$

Usando essa métrica, a Lagrangiana (4.2.3) pode ser colocada na forma (4.1.12).

Para o caso $N=1$ e $G_{\alpha \beta}=1$

$$
\operatorname{det} G_{A B}=-2\left(a_{1} a_{2} a_{3} F(\phi)\right)^{2}\left(F(\phi)+\frac{3}{2}\left(F^{\prime}(\phi)\right)^{2}\right) .
$$

Mais uma vez, este determinante é essencial para o estudo das singularidades geométricas e dinâmicas. Vemos que, além de apresentar a mesma singularidade que surge nos modelos isotrópicos, o modelo anisotrópico tem uma outra singularidade associada a $F(\phi)=0$, que já foi descrita em [32] através de uma extensa análise dinâmica, o que mostra a vantagem de se usar um método mais direto como o apresentado aqui.

\subsection{Modelos $f(R)$}

Fazendo uso da relação de equivalência entre os modelos escalares-tensoriais e $f(R)$, expressa da forma

$$
F=f^{\prime}(\phi) ; \quad V=\phi f^{\prime}-f
$$

podemos aplicar os métodos usados na seção anterior para os modelos $f(R)$ em duas métricas diferentes. Nos dois casos, $G_{\alpha \beta}=0$.

\subsubsection{Métrica isotrópica}

Para um Universo de FLRW com curvatura nula, escrevemos a métrica Lorentziana

$$
G_{A B}(a, \phi)=\left(\begin{array}{cc}
6 a f^{\prime} & 3 a^{2} f^{\prime \prime} \\
3 a^{2} f^{\prime \prime} & 0
\end{array}\right)
$$


que permite escrever a Lagrangiana na forma

$$
L\left(\phi_{A}, \dot{\phi^{A}}\right)=G_{A B} \dot{\phi^{A}} \dot{\phi}^{B}-2 V_{e f f}(\phi)=a^{3}\left[6 f^{\prime} H^{2}+6 f^{\prime \prime} H \dot{\phi}-2 \phi f^{\prime}+2 f\right] .
$$

A Lagrangiana (4.3.3) é equivalente à (3.2.12).

A exigência det $G_{A B} \neq 0$ leva ao aparecimento da singularidade $f^{\prime \prime}=0$, que já surgia anteriormente na definição da superfície de energia (3.0.6) do espaço de fase dos modelos $f(R)$ (ver capítulo 3).

\subsubsection{Métrica anisotrópica}

Para uma métrica anisotrópica de Bianchi em quatro dimensões, a Lagrangiana do modelo $f(R)$ é dada por

$$
L=\left(a_{1} a_{2} a_{3}\right)\left[2 f^{\prime}\left(H_{1} H_{2}+H_{1} H_{3}+H_{2} H_{3}\right)-2 f^{\prime \prime} \dot{\phi}\left(H_{1}+H_{2}+H_{3}\right)-2 \phi f^{\prime}+2 f\right]
$$

A métrica da variedade associada pode ser escrita agora como

$$
G_{A B}\left(a_{1}, a_{2}, a_{3}, \phi\right)=\left(\begin{array}{cccc}
0 & a_{3} f^{\prime} & a_{2} f^{\prime} & a_{2} a_{3} f^{\prime \prime} \\
a_{3} f^{\prime} & 0 & a_{1} f^{\prime} & a_{1} a_{3} f^{\prime \prime} \\
a_{2} f^{\prime} & a_{1} f^{\prime} & 0 & a_{1} a_{2} f^{\prime \prime} \\
a_{2} a_{3} f^{\prime \prime} & a_{1} a_{3} f^{\prime \prime} & a_{1} a_{2} f^{\prime \prime} & 0
\end{array}\right),
$$

que permite escrever (4.3.4) na forma (4.1.12).

Agora a condição det $G_{A B} \neq 0$ leva à singularidade $f^{\prime} f^{\prime \prime}=0$. Isto quer dizer que além da singularidade anterior, $f^{\prime \prime}=0$, surgiu uma nova possibilidade $f^{\prime}=0$, que anteriormente já havia sido determinada a partir da identificação de $f^{\prime}$ com a constante de acoplamento gravitacional de Newton $G$ (ver capítulo 3). Como já mencionado, ela também havia sido detectada em trabalhos que envolviam o uso da métrica de Bianchi em modelos de acoplamento não-mínimo [32], através da análise dinâmica das equações. 


\section{Capítulo 5}

\section{Conclusões e Comentários}

A Cosmologia atual enfrenta problemas que são, até certo ponto, causados pelo seu próprio sucesso e desenvolvimento nos últimos anos. Ela precisa dar conta de fenômenos desconhecidos até o fim do século passado, como a aceleração da expansão do Universo, e deve acomodar teorias bem estabelecidas na descrição da estrutura e evolução do Universo, entre elas a inflação e a nucleossíntese. Por esses motivos, é necessário procurar alternativas aos modelos teóricos que, embora satisfatórios até o advento de observações mais refinadas, não são suficientes para fornecer uma visão cientificamente adequada do Universo.

As teorias de gravidade modificada têm o intuito de suprir as falhas do chamado modelo cosmológico padrão, ao mesmo tempo em que se utilizam do arsenal matemático provido pela Relatividade Geral, uma das teorias de maior êxito da história da ciência.

Mostramos aqui o estudo de modelos escalares-tensoriais a partir do ponto de vista das equações dinâmicas, que permitem investigar o comportamento genérico das soluções das equações de campo sem resolvê-las efetivamente (o que seria muito difícil ou até mesmo impossível). Analisamos o espaço de fase de uma família desses modelos, os do tipo Brans-Dicke, e verificamos o aparecimento de regiões proibidas às órbitas das soluções, relacionadas a valores não permitidos da energia do sistema como estabelecida através do vínculo hamiltoniano. Para uma exploração de uma das possibilidades do modelo, implementamos um potencial do tipo $V(\phi)=\Lambda \phi$, sendo $\Lambda$ a constante cosmológica, ao mesmo tempo tão necessária ao ajuste de dados observacionais e de tão difícil acomodação teórica. Vimos também nesse caso o aparecimento de um espaço de fase com duas folhas e regiões proibidas. Também determinamos serem os pontos fixos soluções tipo de Sitter, ou seja, que levam a uma expansão exponencial do fator 
de escala do Universo.

Em seguida, seguindo na linha da busca por modelos alternativos de gravitação, investigamos os aspectos dinâmicos dos chamados modelos $f(R)$, que não exigem a introdução de um campo escalar. De forma análoga, investigamos seu espaço de fase, composto de apenas uma folha (o que nos permitiu inferir sem esforço a inexistência de comportamento caótico para as órbitas das soluções do sistema dinâmico), e determinamos as regiões proibidas e as soluções de de Sitter. Além disso, escrevemos a Lagrangiana e a Hamiltoniana efetivas para esses modelos, inclusive na sua formulação que permite uma identificação com os modelos escalares-tensoriais.

Essas investigações foram feitas utilizando-se uma métrica de Friedmann-RobertsonWalker-Lemaître, que corresponde em grande medida ao Universo observável em sua isotropia e homogeneidade. Entretanto, é possível que em alguma fase da evolução do Universo a sua expansão se tenha dado de forma não totalmente isotrópica. Existe uma grande variedade de métricas que podem ser utilizadas para estudar um cenário deste tipo. Mostramos aqui como o uso de uma dessas métricas, a de Bianchi tipo I (de fato, a mais simples), aplicada a um modelo escalar-tensorial (já descrito em $[32,115,116])$ com acoplamento não-mínimo entre o campo escalar $\phi$ e o escalar de curvatura $R$ e ao modelo $f(R)$, leva ao aparecimento de singularidades geométricas nesses modelos. Para isso, foi necessário que nos valêssemos do Princípio de MaupertuisJacobi, que relaciona a Lagrangiana de um sistema mecânico a uma geodésica em uma variedade associada, dotada de uma métrica cujo determinante define o aparecimento das singularidades. A utilidade deste método está em sua simplicidade em relação à obtenção das singularidades, que de outra forma seriam acessíveis através de análises dinâmicas mais complicadas.

É desnecessário dizer que o estudo feito aqui não é exaustivo de nenhum dos assuntos abordados. Ao contrário, deve ser visto como ponto de partida para o aprofundamento teórico das técnicas e modelos relevantes para a Cosmologia atual. Como desdobramentos desse estudo podem ser citados: a implementação de modelos escalares-tensoriais ou $f(R)$ mais específicos e realísticos, possivelmente através da utilização de vínculos observacionais ainda por serem obtidos; o estudo do comportamento a campo fraco (o limite Newtoniano) desses modelos, que vai permitir estabelecer sua validade no âmbito do Sistema Solar, por exemplo, e na sua aplicação a problemas do tipo colapso estelar; o uso de outras métricas e formalismos que simplifiquem as análises e/ou tenham maior significado físico; a inclusão da matéria, com uma possível interação entre matéria escura 
e energia escura.

Muitos são os desafios da Cosmologia para os próximos anos e muitos também serão os avanços observacionais que serão obtidos em breve com os lançamentos de novas sondas e a construção de novos telescópios. Certamente, uma nova geração de cientistas estará debruçada sobre uma grande quantidade de dados mais precisos na busca incessante por uma descrição melhor do Universo em que vivemos. 


\section{Apêndice A}

\section{Método de Lyapunov}

Há uma vasta literatura apresentando análises do espaço de fase das equações dinâmicas para o modelo de Brans-Dicke através do método de linearização, e.g., [60, 70, 71]. Este método, embora bastante útil numa grande variedade de casos, pode apresentar inconvenientes e limitações.

Uma maneira mais poderosa de se analisar as características do espaço de fase de um sistema dinâmico de equações, no âmbito de modelos cosmológicos, é o chamado método direto de Lyapunov. Este método permite uma investigação mais ampla das soluções possíveis para as equações, com base em aspectos do espaço de fase tais como existência e localização dos pontos fixos do modelo, estabilidade e sensibilidade dessas soluções às condições iniciais.

O método parte da busca pela chamada função de Lyapunov $\mathcal{L}$ do modelo, que geralmente é obtida a partir de uma combinação da energia do sistema. Essa função deve preencher os seguintes requisitos:

1. $\mathcal{L}(x)$ deve ser contínua em uma certa região $\Omega$ ao redor da origem (bem como suas primeiras derivadas parciais).

2. $\mathcal{L}(0)=0$.

3. $\mathcal{L}(x)>0$ fora da origem.

De posse da função que satisfaz os requisitos anteriores, e que é chamada de candidata a função de Lyapunov, podemos estudar a estabilidade dos pontos fixos do sistema. Os teoremas de estabilidade de Lyapunov estabelecem para os pontos fixos: 


\section{Estabilidade}

Se existe uma função de Lyapunov $\mathcal{L}(x)$ para a qual $\dot{\mathcal{L}}(x) \leq 0$ na vizinhança da origem, então a origem é estável.

\section{Estabilidade assintótica}

Se $-\dot{\mathcal{L}}$ também é positiva na região $\Omega$, então a estabilidade é assintótica.

\section{Instabilidade}

Se $\dot{\mathcal{L}}>0$, então a origem é instável.

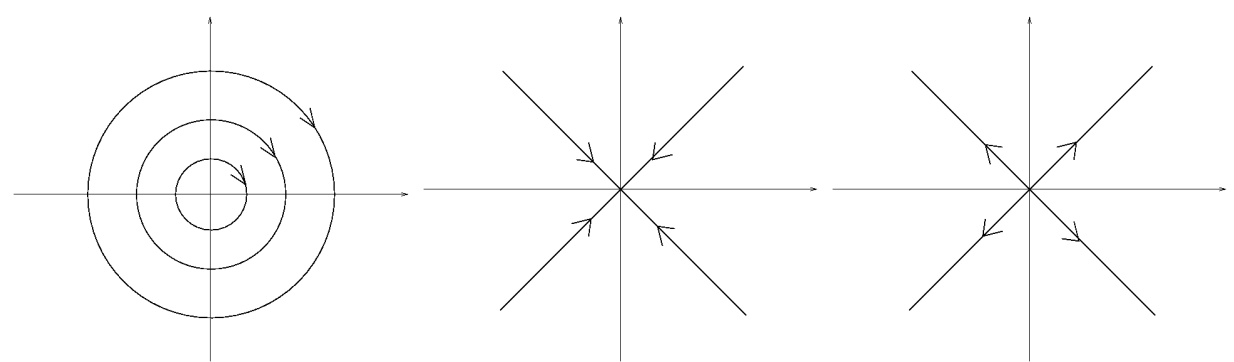

Figura A.1: Exemplos de sistemas dinâmicos cujas origens são, respectivamente, um ponto estável, assintoticamente estável e instável.

A figura A.1 exemplifica as três situações consideradas.

Por definição, se a origem é um ponto de estabilidade assintótica, então existe uma vizinhança $N$ da origem de tal forma que qualquer curva que seja solução do sistema dinâmico e que comece em $N$ tende à origem. A união de todas essas curvas que tendem ao ponto assintoticamente estável é chamada de bacia de atração. O tamanho da bacia indica a perturbação máxima que o sistema pode sofrer e ainda assim retornar ao equilíbrio.

Os detalhes formais do método, como a demonstração dos teoremas, não serão explicitados aqui, podendo ser encontrados em vários textos. As referências [16]-[19] são alguns exemplos de fontes para um estudo mais aprofundado. 


\section{Apêndice B}

\section{As equações de Friedmann com constante cosmológica}

Em um Universo homogêneo e isotrópico em que as distâncias são definidas pela métrica de Friedmann-Lemaître-Robertson-Walker

$$
\mathrm{d} s^{2}=-\mathrm{d} t^{2}+a^{2}(t)\left[\frac{\mathrm{d} r^{2}}{1-K r^{2}}+r^{2}\left(\mathrm{~d} \theta^{2}+\sin ^{2} \theta \mathrm{d} \varphi^{2}\right)\right]
$$

onde $a$ é o fator de escala que determina a taxa de variação das distâncias entre dois pontos do espaço e $K$ é o parâmetro de curvatura espacial ( $K$ vale $+1,0$ ou -1, respectivamente, para Universos fechados, planos ou abertos), e dotado de uma constante cosmológica positiva $\Lambda$, as equações de Einstein se escrevem da forma (fazendo $G=c=1$ )

$$
R_{\mu \nu}-\frac{1}{2} R g_{\mu \nu}+\Lambda g_{\mu \nu}=8 \pi T_{\mu \nu}
$$

Aqui, $T_{\mu \nu}$ é o tensor de energia-momento. As eqs. (B.0.2) dão origem às equações de Friedmann

$$
H^{2}=\left(\frac{\dot{a}}{a}\right)^{2}=\frac{8 \pi}{3} \rho+\frac{\Lambda}{3}-\frac{K}{a^{2}}
$$

e

$$
\frac{\ddot{a}}{a}=-\frac{4 \pi}{3}(\rho+3 p)+\frac{\Lambda}{3},
$$

onde definimos a quantidade $H=\dot{a} / a$, o parâmetro de Hubble, que na verdade é um observável da teoria, já que o fator de escala obedece à relação

$$
a=\frac{1}{1+z}
$$


com o redshift $z$ de uma fonte luminosa em movimento em relação ao observador. Observações astronômicas muito freqüentemente utilizam-se do redshift para a determinação de distâncias.

É útil fazer $K=0$ e $\Lambda=0$ na eq. (B.0.3) e definir a densidade crítica

$$
\rho_{c}=\frac{3 H^{2}}{8 \pi}
$$

de forma que agora se pode representar as densidades de energia de cada uma das componentes $i$ do Universo como a razão

$$
\Omega_{i}=\frac{\rho_{i}}{\rho_{c}}
$$

$\Omega_{i}$ é o parâmetro de densidade para a componente $i$. O parâmetro de densidade total é simplesmente a soma

$$
\Omega=\sum_{i} \Omega_{i}
$$

envolvendo, por exemplo, as componentes de radiação $\Omega_{r}$, de matéria bariônica $\Omega_{b}$, de matéria escura $\Omega_{D M}$, de curvatura $\Omega_{K}$ e de constante cosmológica $\Omega_{\Lambda}$, entre outras que se queira considerar para o fluido cósmico. É comum levar-se em conta apenas a componente que domina uma determinada fase da evolução do Universo, e desprezar-se as demais.

A densidade crítica é representada por $\Omega_{c}=1$. Observações recentes indicam que o Universo atual está numa fase muito próxima àquela caracterizada por apresentar o valor exato da densidade crítica, reforçando a noção de que, em larga escala, a seção espacial do espaço-tempo é plana. 


\section{Bibliografia}

[1] R. M. Wald, "General Relativity". The University of Chicago Press (1984).

[2] P. J. E. Peebles, "Principles of Physical Cosmology". Princeton University Press (1993).

[3] R. E. de Souza, "Introdução à Cosmologia". Edusp (2004).

[4] M. S. Turner e E. W. Kolb, "The Early Universe". Addison-Wesley (1990).

[5] S. W. Hawking e G. F. R. Ellis, "The Large Scale Structure of Space-Time". Cambridge University Press (1973).

[6] T. Padmanabhan, "Cosmology and Astrophysics Through Problems". Cambridge University Press (1996).

[7] R. C. Tolman, "Relativity Thermodynamics and Cosmology". Dover Publications (1987).

[8] J. L. Synge e A. Schild, "Tensor Calculus". Dover Publications (1978).

[9] J. L. Synge, "Relativity: the General Theory". North-Holland Publishing Company (1971). Publications (1978).

[10] B. R. Iyer, N. Mukunda e C. V. Vishveshwara (ed.), "Gravitation, Gauge Theories and the Early Universe". Kluwer Academic Publishers (1989).

[11] V. Faraoni, "Cosmology in Scalar-Tensor Theory". Kluwer Academic Publishers (2004).

[12] S. Weinberg, "Gravitation and Cosmology - Principles and Applications of the General Theory of Relativity". John Wiley \& Sons (1972). 
[13] C. W. Misner, Kip. S. Thorne e J. A. Wheeler, "Gravitation", W. H. Freeman and Company (1973).

[14] C. M. Will, "Theory and Experiment in Gravitational Physics". Cambridge University Press (1993).

[15] A. D. Linde, "Inflation and Quantum Cosmology". Academic Press (1990).

[16] J. LaSalle e S. Lefschetz, "Stability by Lyapunov's Direct Method". Academic Press (1967).

[17] D. Merkin, "Introduction to the Theory of Stability". Springer (1997).

[18] M. W. Hirsch e S. Smale, "Differential Equations, Dynamical Systems, and Linear Algebra". Academic Press (1974).

[19] J. W. Walker, "Dynamical Systems and Evolution Equations: Theory and Applications". Plenum Press (1980).

[20] H. Goldstein, "Classical Mechanics". Addison-Wesley (1980).

[21] M. S. Turner, "Inflation: From Theory To Observation and Back", Annals N.Y. Acad. Sci. 759 (1995) 153 [astro-ph/9703194]

[22] M. S. Turner e L. M. Krauss, "The Cosmological Constant is Back", Gen. Rel. Grav. 27 (1995)1137 [astro-ph/9504003]

[23] W. Hu, N. Sugiyama e J. Silk, "The Physics of Microwave Background Anisotropies", Nature 386(1997) 37-43

[24] W. Hu, "Ringing in the New Cosmology", Nature 404 (2000) 939-940

[25] S. Perlmutter et al., "Measurements of $\Omega$ and $\Lambda$ from 42 High-Redshift Supernovae", Astrophys. J. 517 (1998) 565-586

[26] A. G. Riess et al., "Observational Evidence from Supernovae for an Accelerating Universe and a Cosmological Constant", Astronom. J. 116 (1998) 1009-1038

[27] L.M. Díaz-Rivera e L.O. Pimentel, "Cosmological Models with Dynamical $\Lambda$ in Scalar-Tensor Theories", Phys. Rev. D 60 (1999) 123501 
[28] S. Perlmutter et al., "Discovery of a Supernova Explosion at Half the Age of the Universe", Nature 391(1998) 51-54

[29] P. de Bernardis et al., "A Flat Universe from High-Resolution Maps of the Cosmic Microwave Background Radiation", Nature 404(2000) 955-959

[30] C. Brans e R. H. Dicke, "Mach's Principle and a Relativistic Theory of Gravitation", Phys. Rev. 124 (1961) 925-935

[31] G. Esposito-Farèse e D. Polarski, "Scalar-Tensor Gravity in an Accelerating Universe", Phys. Rev. D 63 (2001) 063504

[32] A. Saa et al., "Superinflation, Quintessence, and Nonsingular Cosmologies", Phys. Rev. D63, 067301 (2001); "Superinflation, Quintessence, and the Avoidance Of The Initial Singularity", Int. J. Theor. Phys. 40, 2295 (2001); L.R. Abramo, L. Brenig, E. Gunzig, A. Saa, "On the Singularities of Gravity in the Presence of Nonminimally Coupled Scalar Fields", Phys. Rev. D67, 027301 (2003); "Dynamical Study of the Singularities of Gravity in the Presence of Nonminimally Coupled Scalar Fields",Int. J. Theor. Phys. 42 (2003) 1145 [gr-qc/0305008].

[33] H. Kodama e M. Sasaki, "Cosmological Perturbation Theory", Prog. Theor. Phys. Suppl. 78 (1984)

[34] V. F. Mukhanov, H. A. Feldman e R. H. Brandenberger, "Theory of Cosmological Perturbations", Phys. Rep. 5 \& 6 (1992) 203-333

[35] K. A. Malik e D. Sands, "Gauge-Invariant Variables on Cosmological Hypersurfaces", gr-qc/9804046

[36] X. Chen e M. Kamionkowski, "Cosmic Microwave Background Temperature and Polarization Anisotropy in Brans-Dicke Cosmology", Phys. Rev. D 60 (1999) 104036 [astro-ph/9905368]

[37] T. Chiba, N. Sugiyama e J. Yokoyama, "Imprints of the Metrically Coupled Dilaton on Density Perturbations in Inflationary Cosmology", Nucl. Phys. B 530 (1998) 304-324

[38] J. D. Barrow e J. P. Mimoso, "Perfect Fluid Scalar-Tensor Cosmologies", Phys. Rev. D 50(6) (1994) 3746-3754 
[39] J. E. Lidsey et al., "Reconstructing the Inflaton Potential - An Overview", Rev. Mod. Phys. 69 (1997) 373-410

[40] T. Hirai e K. Maeda, "Gauge-Invariant Cosmological Perturbations in Generalized Einstein Theories", Astrophys. J. 431 (1994) 6-19

[41] J. Hwang, "Cosmological Perturbations in Generalised Gravity Theories: Formulation", Class. Quantum Grav. 7 (1990) 1613-1631

[42] J. Hwang, "Perturbations of the Robertson-Walker Space: Multicomponent Sources and Generalized Gravity", Astrophys. J., 375 (1991) 443-462

[43] J. Hwang, "Conserved Variable in the Perturbed Hydrodynamic World Model", Phys. Rev. D 60 (1999) 103512

[44] B. Bertotti, L. Iess e P. Tortora, "A Test of General Relativity Using Radio Links with the Cassini Spacecraft", Nature 425 (2003) 374-376

[45] H. Kim, "Brans-Dicke Scalar Field as a Unique k-essence", Phys. Lett. B 606 (2005) 223-233

[46] H. Kim, "Brans-Dicke Theory as an Unified Model for Dark Matter-Dark Energy", Mon. Not. Roy. Astron. Soc. 364 (2005) [astro-ph/0408577]

[47] C. Armendariz-Picon, V. Mukhanov e P. J. Steinhardt, "Essentials of $k$-essence", Phys. Rev. D 63 (2001) 103510

[48] G. Esposito-Farèse, "Tests of Scalar-Tensor Gravity", AIP Conf.Proc. 736 (2004) 35 [gr-qc/0409081]

[49] V. Acquaviva, C. Baccigalupi, S. M. Leach, A. R. Liddle e F. Perrotta, "Structure Formation Constraints on the Jordan-Brans-Dicke Theory", Phys. Rev. D 71 (2005) 104025

[50] A. R. Liddle, A. Mazumdar e J. D. Barrow, "Radiation-matter Transition in Jordan-Brans-Dicke Theory", Phys. Rev. D 58 (1998) 027302

[51] R.J. Scherrer, "Purely Kinetic k-essence as Unified Dark Matter", Phys. Rev. D 93 (2004) 011301 
[52] M. Malquarti, E. J. Copeland e A. R. Liddle, " $k$-essence and the Coincidence Problem", Phys. Rev. D 68 (2003) 023512

[53] C. H. Brans, "The Roots of Scalar-Tensor Theory: an Approximate History", grqc/0506063

[54] V. Faraoni, "Phase Space Geometry in Scalar-Tensor Cosmology", Ann. Phys. 317 (2005) 366

[55] V. Faraoni, "Modified Gravity and the Stability of de Sitter Space", Phys. Rev. D 72 (2005) 061501

[56] V. Faraoni, "Negative Energy and Stability in Scalar-Tensor Gravity", Phys. Rev. D 70 (2004) 081501(R)

[57] V. Faraoni, "Illusions of General Relativity in Brans-Dicke Gravity", Phys. Rev. D 59 (1999) 084021

[58] V. Faraoni, "Einstein Frame or Jordan Frame?", Int.J.Theor.Phys 38 (1999) 217225

[59] D. F. Torres, "Quintessence, Superquintessence, and Observable Quantities in Brans-Dicke and Nonminimally Coupled Theories", Phys. Rev. D 66 (2002) 043522

[60] C. Santos e Ruth Gregory, "Cosmology in Brans-Dicke Theory with a Scalar Potential", Ann. Phys. 258 (1997) 111

[61] G. Huey e B. D. Wandelt, "Interacting Quintessence, the Coincidence Problem and Cosmic Acceleration", Phys. Rev. D 74 (2006) 023519 [astro-ph/0407196]

[62] F. C. Carvalho e A. Saa, "Non-minimal Coupling, Exponential Potentials and the $w<-1$ Regime of Dark Energy", Phys. Rev. D 70 (2004) 087302

[63] F. C. Carvalho, J. S. Alcaniz, J. A. S. Lima e R. Silva, " Scalar-Field-Dominated Cosmology with a Transient Accelerating Phase", Phys. Rev. Lett. 97 (2006) 081301

[64] F. C. Carvalho, "Teorias Escalares-Tensoriais e o Regime Superacelerado do Universo: Aspectos Dinâmicos", tese de doutorado, IFUSP (2005). 
[65] D. La e P. Steinhardt, "Extended Inflationary Cosmology", Phys. Rev. Lett. 62 (1989) 376

[66] E. J. Weinberg, "Some Problems With Extended Inflation", Phys. Rev. D 40 (1989) 3950

[67] T. Damour e K. Nordtvedt, "General Relativity as a Cosmological Attractor of Scalar-Tensor Theories", Phys. Rev. Lett. 70 (1993) 2217

[68] T. Damour e K. Nordtvedt, "Tensor-Scalar Cosmological Models and their Relaxation Toward General Relativity", Phys. Rev. D 48 (1993) 3436

[69] P. Steinhardt e F. Accetta, "Hyperextended Inflation", Phys. Rev. Lett. 64 (1990) 2740

[70] S. J. Kolitch e D. M. Eardley, "Qualitative Analysis of Brans-Dicke Universes with a Cosmological Constant", Ann. Phys. 246 (1996) 121

[71] S. J. Kolitch, "Behavior of Friedmann-Robertson-Walker Cosmological Models in Scalar-Tensor Gravity", Ann. Phys. 241 (1995) 128

[72] A. G. Sanchez et al., "Cosmological Parameters from CMB Measurements and the Final 2dFGRS Power Spectrum", Mon. Not. Roy. Astron. Soc. 366 (2006) 189 [astro-ph/0507583]

[73] S. M. Carroll, M. Hoffman e M. Trodden, "Can the Dark Energy Equation-of-State Parameter $w$ be Less Than -1?", Phys. Rev. D 68 (2003) 023509

[74] R. R. Caldwell, M. Kamionkowski e N. N.Weinberg , "Phantom Energy: Dark Energy with $w<-1$ Causes a Cosmic Doomsday", Phys. Rev. Lett. 91 (2003) 071301

[75] R. R. Caldwell e E. Linder, "Limits of Quintessence", Phys. Rev. Lett. 95 (2005) 141301

[76] H. K. Jassal, J. S. Bagla e T. Padmanabhan, "Observational Constraints on Low Redshift Evolution of Dark Energy: How Consistent are Different Observations?", Phys. Rev. D 72 (2005) 103503 
[77] L. Perivolaropoulos, "Constraints on Linear-Negative Potentials in Quintessence and Phantom Models from Recent Supernova Data", Phys. Rev. D 71 (2005) 063503

[78] A. Lue e G. D. Starkman, "How a Brane Cosmological Constant can Trick us into Thinking that $w<-1 "$, Phys. Rev. D 70 (2004) 101501 [astro-ph/0408246]

[79] Website do Observatório Espacial de Raios X Chandra: http://chandra.harvard.edu/resources/illustrations.html

[80] J. C. C. de Souza e A. Saa, Braz. J. Phys., "Phase Space Solutions in Scalar-Tensor Cosmological Models", Braz. J. Phys.35, no. 4B, (2005) 1041 .

[81] A.G. Riess et al., "Type Ia Supernova Discoveries at $z>1$ From the Hubble Space Telescope: Evidence for Past Deceleration and Constraints on Dark Energy Evolution". Astrophys. J 607 (2004) 665 [astro-ph/0402512]

[82] SNLS, The Canada-France-Hawaii Telescope Legacy Survey Supernova Program, ver

http://www.cfht.hawaii.edu/SNLS/

[83] S. Perlmutter et al., "Measurements of the Cosmological Parameters Omega and Lambda From the First 7 Supernovae at $z \geq 0.35 "$. ApJ 483 (1997) 565

[84] P.J. Steinhardt, "A Quintessential Introduction to Dark Energy". Phil. Trans. Roy. Soc. Lond. A361 (2003) 2497.

[85] Satélite WMAP (Wilkinson Microwave Anisotropy Probe), ver http: //lambda.gsfc.nasa.gov/

[86] Satélite SNAP (Supernova/Acceleration Probe) Satellite, ver http://snap.lbl.gov/

[87] S. M. Carroll, "The Cosmological Constant", Living Rev. Relativity 4 (2001) 1. URL:

http://www . livingreviews . org/lrr-2001-1

[88] R. R. Caldwell, R. Dave e P.J. Steinhardt, "Cosmological Imprint of an Energy Component with General Equation of State", Phys. Rev. Lett. 80 (1998) 1582 
[89] V. Faraoni, "Superquintessence", Int. J. Mod. Phys. D11 (2002) 471

[90] E. Gunzig, V. Faraoni, A. Figueiredo, T.M. Rocha e L. Brenig, "The Dynamical System Approach to Scalar Field Cosmology", Class. Quantum Grav. 17 (2000) 1783

[91] V. Faraoni, "De Sitter Attractors in Generalized Gravity", Phys. Rev. D 70 (2004) 044037

[92] V. Faraoni e W. Israel, "Dark Energy, Wormholes, and the Big Rip", Phys. Rev. D 71 (2005) 064017

[93] V. Faraoni e M. N. Jensen, "Non-Chaotic Dynamics in General-Relativistic and Scalar-Tensor Cosmology", Class. Quantum Grav. 23 (2006) 4215

[94] V. Faraoni e M. N. Jensen, "Extended Quintessence, Inflation, and Stable de Sitter Spaces", Class. Quantum Grav. 23 (2006) 3005

[95] V. Faraoni e S. Nadeau, "Stability of Modified Gravity Models", Phys. Rev. D 72 (2005) 124005

[96] V. Faraoni e S. Nadeau, "The (Pseudo)Issue of the Conformal Frame Revisited", Phys. Rev. D 75 (2007) 023501

[97] V. Faraoni, "Solar System Experiments do Not Yet Veto Modified Gravity Models", Phys. Rev. D 74 (2006) 023529

[98] V. Faraoni, "Matter Instability in Modified Gravity", Phys. Rev. D 74 (2006) 104017 [astro-ph/0610734]

[99] T. Chiba, T. L. Smith e A. L. Erickcek, "Solar System Constraints to General $f(R)$ Gravity", Phys. Rev. D 75 (2007)124014 [astro-ph/0611867]

[100] G. J. Olmo, "Post-Newtonian Constraints on $f(R)$ Cosmologies in Metric and Palatini Formalism", Phys. Rev. D 72 (2005) 083505

[101] L. Amendola, R. Gannouji, D. Polarski e S. Tsujikawa , "Conditions for the Cosmological Viability of $f(R)$ Dark Energy Models", Phys. Rev. D 75 (2007) 083504 [gr-qc/0612180] 
[102] P. Teyssandier e P. Tourrenc, "The Cauchy Problem for the $R+R^{2}$ Theories of Gravity Without Torsion", J. Math. Phys 24 (1983) 2793

[103] D. Wands, "Extended Gravity Theories and the Einstein-Hilbert Action", Class. Quant. Grav. 11 (1994) 269

[104] T. Chiba, "1/R Gravity and Scalar-Tensor Gravity", Phys. Lett. B 575 (2003) 1

[105] L.R. Abramo, L. Brenig e E. Gunzig, "On the Stability of Gravity in the Presence of an NMC Scalar Field", Phys. Lett. B 549 (2002) 13

[106] T. P. Sotiriou, " $f(R)$ Gravity and Scalar-Tensor Theory", Class. Quant. Grav. 23 (2006) 5117

[107] T. P. Sotiriou e S. Liberati, "Metric-Affine $f(R)$ Theories of Gravity", Annals Phys. $322(2007) 935$

[108] A. D. Dolgov e M. Kawasaki, "Can Modified Gravity Explain Accelerated Cosmic Expansion?", Phys. Lett. B $\mathbf{5 7 6 3}$ (2003) 1

[109] Nojiri S and Odintsov S D 2003, Phys. Rev. D 68 123512; 2004, Gen. Rel. Grav. 36, 1765; 2006, Phys. Rev. D 74086005

[110] J. D. Barrow e A. Ottewill, "The Stability of General Relativistic Cosmological Theory" J. Phys. A 16 (1983) 2757

[111] J.C.C. de Souza e V. Faraoni, "The Phase Space View of $f(R)$ Gravity". Class. Quantum Grav. 24 (2007) 3637

[112] S. Capozziello, S. Nesseris e L. Perivolaropoulos, "Reconstruction of the ScalarTensor Lagrangian from a LCDM Background and Noether Symmetry", [arXiv:0705.3586]

[113] Y. Fujii e M. Sasaki, "Gravitational scalar field coupled directly to the Maxwell Field and its Effect to Solar-System Experiments", Phys. Rev. D 75 (2007) 064028

[114] P. K. Townsend e M. N. R. Wohlfarth, "Cosmology as a Geodesic Motion", Class. Quant. Grav. 21 (2004) 5375 
[115] L. A. Elias e A. Saa, "Homogeneous Cosmologies and the Maupertuis-Jacobi Principle", Phys. Rev. D 75 (2007) 107301

[116] L. A. Elias e A. Saa, "Non-Minimally Coupled Cosmology as Geodesic Motion", PoS IC2006 (2006) 039 